\title{
Le site Bronze ancien de La Caillouerie à Saint-Lô d'Ourville (50), un site spécialisé en contexte littoral?
}

The early Bronze Age settlement of "La Caillouerie" in Saint-Lô d'Ourville (50), a specialized site in seashore environment?

Der älterbronzezeitliche Fundplatz "La Caillouerie" von Saint-Lô d'Ourville (Manche), ein spezialisierter Fundplatz im Küstenbereich?

El sitio de la Edad de Bronce Antigua de "La Caillouerie" en Saint-Lô-d'Ourville (Manche): un sitio especializado en contexto costero?

Lolita Rousseau, Cyrille Billard, François Charraud, Gilles Laisné et Gérard Vilgrain-Bazin

\section{(2) OpenEdition}

\section{Journals}

Édition électronique

URL : https://journals.openedition.org/rao/2479

DOI : $10.4000 /$ rao. 2479

ISBN : 978-2-7535-4053-8

ISSN : $1775-3732$

Éditeur

Presses universitaires de Rennes

Édition imprimée

Date de publication : 15 décembre 2014

Pagination : $37-55$

ISBN : $978-2-7535-4051-4$

ISSN : 0767-709X

Référence électronique

Lolita Rousseau, Cyrille Billard, François Charraud, Gilles Laisné et Gérard Vilgrain-Bazin, « Le site Bronze ancien de La Caillouerie à Saint-Lô d'Ourville (50), un site spécialisé en contexte littoral ?», Revue archéologique de l'Ouest [En ligne], 31 | 2014, mis en ligne le 15 décembre 2016, consulté le 22 août 2022. URL : http://journals.openedition.org/rao/2479; DOI : https://doi.org/10.4000/rao.2479 


\title{
Le site Bronze ancien de La Caillouerie à Saint-Lô d'Ourville (50), un site spécialisé en contexte littoral?
}

\author{
The Early Bronze Age Settlement of "La Caillouerie" in Saint-Lô d'Ourville (50), \\ a Specialized Site in Seashore Environment?
}

\author{
Lolita Rousseau* \\ avec la collaboration de Cyrille Billard**, François Charraud ${ }^{* * *}$, \\ Gilles LaISnÉ ${ }^{* * * *}$ et Gérard VILGRAIN-BaZIN ${ }^{* * * *}$
}

\begin{abstract}
Résumé : Le site de " la Caillouerie " à Saint-Lô d'Ourville, localisé au sein du havre de Portbail, a été découvert fortuitement suite à l'érosion intensive de la plage dans les années 1990/2000. Daté du début de l'âge du Bronze ancien, il a livré une quantité très importante de mobilier lithique sur une surface restreinte. L'étude de cette série a permis de mettre en évidence un atelier de débitage de petits galets côtiers par percussion posée sur enclume. La présence de nombreux artefacts pouvant correspondre typologiquement à des pièces esquillées, associés à des éléments fauniques fracturés intentionnellement nous a conduit à réaliser une expertise tracéologique afin de valider ou non l'hypothèse d'un lien entre ces différents éléments. Cette étude a permis de montrer que seule une partie de ces pièces avait effectivement été utilisée comme pièces intermédiaires pour fendre des os longs. Ce site permet d'apporter de nouvelles connaissances au sujet des productions lithiques du début de l'âge du Bronze, et de s'interroger sur leur place dans l'exploitation de l'environnement littoral. Il permet également de proposer l'hypothèse de la présence d'ateliers spécialisés probablement en retrait de la sphère domestique.
\end{abstract}

Abstract: The site of "La Caillouerie" in Saint-Lô-d'Ourville, discovered after beach erosion, during the 1990'-2000's, is located in Portbail harbor.
Dated to the beginning of the Early Bronze Age, it is characterized by a high concentration of lithic artifacts contained within a small area. Its study
has highlighted a knapping workshop of small coastal pebbles that uses bipolar flaking. Traceological analysis has been used to validate or invalidate the
hypothesis of a link between the presence of artifacts such as splintered pieces and intentionally fractured faunal elements. The results show that only a
portion of these pieces had actually been used as spacers to split the long bones. The site provides new evidence about Early Bronze Age lithic productions,
as well as questioning its role in the exploitation of the coastal environment. The hypothesis of the presence of specialized workshops probably outside of
the domestic sphere will also be discussed in this paper.

Mots clés : âge du Bronze ancien, industrie lithique, amas de débitage, silex, pièces esquillées, percussion sur enclume, galets côtiers, tracéologie, littoral, Manche, os fendus, céramique à anse arciforme.

Keywords: Early Bronze Age, lithic industry, débitage area, fint, splintered pieces, bipolar flaking, coastal pebbles, traceological analysis, seashore, Channel, bones split, horseshoe handle pottery.

\footnotetext{
*Université de Nantes, UMR 6566, LARA; lolita.rousseau@univ-nantes.fr

** DRAC-SRA de Basse-Normandie, Caen.

*** Université Nice Sophia Antipolis, UMR 7264, CEPAM.

**** GRAC (Groupe de Recherches Archéologiques du Cotentin).
} 


\section{INTRODUCTION}

Longtemps, le mobilier lithique du début de l'âge du Bronze n'a pas attiré l'attention des chercheurs, à la fois délaissé par les protohistoriens - car peu sensibilisés à ce type de production - et par les préhistoriens à cause de son aspect peu " attractif » du fait de sa mise en œuvre sommaire, mais aussi parfois ignoré, car souvent hâtivement considéré comme résultant de "pollutions néolithiques » (pièces remaniées, mélanges post-dépositionnels). Seules les armatures de flèches issues de contextes funéraires ont provoqué une certaine curiosité dès la fin du XIX ${ }^{e}$ siècle et ont donné lieu à plusieurs travaux de recherche (Loth, 1908; Mortillet, 1920; Briard, 1970, Nicolas, 2012; etc.). Bien que dès les années 1920 certains auteurs aient soupçonné la persistance des productions lithiques domestiques à l'âge du Bronze (Philippe, 1927; Gardez, 1935; Briard et L'Helgouach, 1957 ; etc.), il faudra attendre les années 1980 pour que le lithique prenne timidement place au sein des ouvrages généraux. Enfin, depuis une quinzaine d'années, ces thématiques sont réexaminées avec un oil neuf, notamment par le biais de l'archéologie préventive et des fouilles sur les sites d'habitat, qui ont permis de mettre en évidence des lots lithiques en cohérence stratigraphique avec les occupations protohistoriques associées. On peut notamment signaler les sites de "Tatihou » (Marcigny et Ghesquière, 2003), île de Molène "Beg ar Loued " (Pailler et al., 2011), ou encore Dolus-d'Oléron " gisement 18 de la Passe de l'Écuissière " (Laroche, 2012), dont les séries lithiques sont encore partiellement inédites pour certains.

Une majorité des sites d'habitats du début de l'âge du Bronze se caractérise par des assemblages lithiques quantitativement faibles (quelques dizaines, voire centaines de pièces tout au plus) et des chaînes opératoires segmentées, bien que quelques exceptions apparaissent avec des sites comportant une production lithique conséquente et aux chaînes opératoires complètes, mais courtes. Cette dichotomie a d'ailleurs été observée au sein des sites Bronze ancien du bassin rhodanien (Bailly, 2002). Le site littoral de "La Caillouerie", daté du début de l'âge du Bronze ancien, semble faire partie de cette seconde singularité. L'étude de son important lot lithique (plus de 10000 pièces, esquilles comprises) permet d'appréhender une facette nouvelle de cette période chronologique, dans le Nord-Ouest de la France : la présence de sites d'activités spécialisées, probablement déconnectés de la sphère domestique.

\section{Présentation du site}

Le site de "La Caillouerie » à Saint-Lô d'Ourville (Manche) se situe au sud-ouest du havre de Portbail, sur l'estran bordant les " dunes de Lindbergh " (fig. 1).

Suite à l'érosion intensive de la plage du fait de son contact direct avec la rivière la Grise et aux importantes précipitations qui ont eu lieu durant l'hiver 1999/2000, une première concentration de mobilier archéologique (lithique et faune) est apparue sur le site. Cette dernière a fait l'objet d'un ramassage de surface par Gilles Laisné et d'un suivi régulier. L'apparition d'une seconde concentration de mobilier archéologique, située à une quarantaine de mètres de la première, a poussé le GRAC (Groupe de recherches archéologiques du Cotentin) à effectuer une petite opération de sauvetage en 2002/2003, sur une surface d'une dizaine de mètres carrés. Cette fouille a permis de mettre en évidence un amas de débitage associé à de la faune (cervidé1). Depuis sa découverte, des sondages mécaniques ont permis de définir la stratigraphie générale du site et de son environnement proche, ainsi que la réalisation de prélèvements visant à la réalisation de datations par le radiocarbone.

\section{DonnÉES STRATIGRAPHIQUES ET CHRONOLOGIQUES}

Dans le cadre du projet collectif de recherche sur "L'exploitation des milieux littoraux en Basse-Normandie ", une coupe stratigraphique des formations superficielles $\mathrm{du}$ havre de Portbail a été relevée sur plus d'un kilomètre de long et assortie d'un ensemble de datations par le radiocarbone (Billard et al., 2006; fig. 2). Elle permet de comprendre l'évolution chronologique, géomorphologique et archéologique du havre, en montrant que ce dernier faisait place à un vaste marais palustre protégé par un cordon littoral, avant d'être envahi par la mer suite à la rupture de ce dernier aux alentours de 2000 av. J.-C. Ce phénomène majeur, bien daté, est d'ailleurs attesté sur d'autres sites de la façade occidentale du département de la Manche, pour la même période. C'est notamment le cas pour le havre de la Vanlée à Lingreville (Billard et al., 1995), pour le site de Saint-Jean-le-Thomas "La plage de Pignochet " (L'Homer, 1995; Billard et al., sous presse), ou encore dans le centre de Granville (Delahaye, 2007). Ce secteur a par ailleurs donné lieu à des observations dans le cadre d'une thèse de doctorat, en suivant une problématique centrée sur l'enregistrement des massifs dunaires (Meurisse, 2007). Ce travail signale

1. Détermination par Lisandre Bedault (UMR 8215, Trajectoires), d'après photographies. 


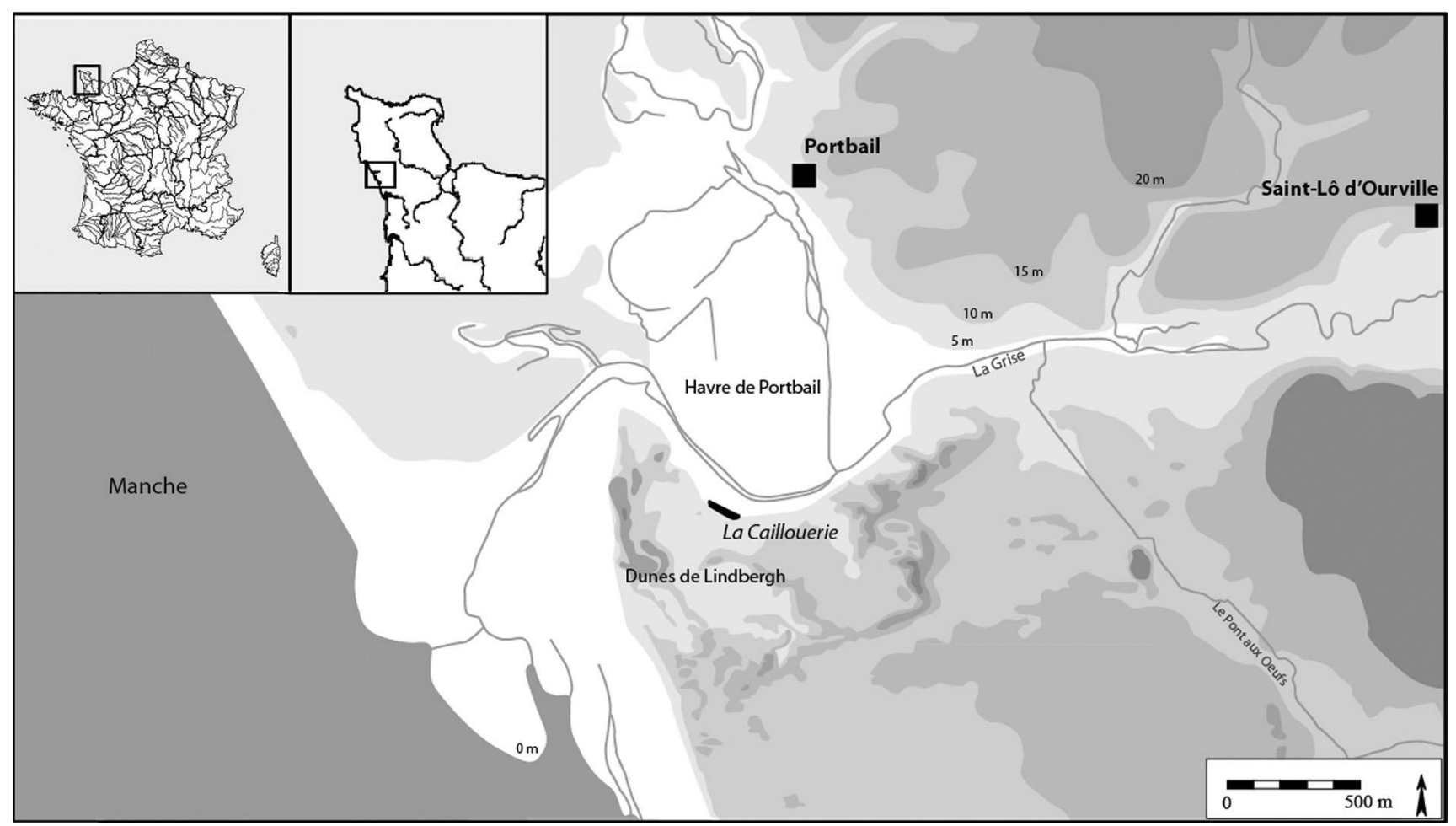

Figure 1 : Saint-Lô d'Ourville "La Caillouerie » : localisation topographique du site. DAO L. Rousseau.

Figure 1: Saint-Lô d'Ourville "La Caillouerie": topographical location of the site. CAD L. Rousseau.

l'impact, jusque-là négligé, des tempêtes sur l'évolution littorale dès 2500 av. J.-C., qui implique la mise en place des premiers cordons dunaires, ainsi que plusieurs épisodes de variations eustatiques (ibid.; Sorrel et al., 2012).

Ce relevé, combiné aux observations de terrain, a permis de replacer le mobilier archéologique dans son contexte chrono-stratigraphique. L'assemblage lithique, protégé par le sable dunaire, reposait sur une couche de sable orangé légèrement induré, d'une puissance de $40 \mathrm{~cm}$ maximum, surmontant le cordon de galets. La présence de faune a permis de dater le niveau archéologique de 2140-1914 cal. BC (Ly-2109). Cette datation peut être comparée à celle correspondant à la fin de la séquence palustre (Ly-13090), dont le remplissage est similaire à celui de la dépression dans laquelle se trouvaient 25 tessons céramiques. L'intégralité de ces tessons, découverts en place, provient d'un vase à cordon préoral et à élément de préhension arciforme (Tableau 1-fig. 3; Billard et al., 2006).

Si ce ne sont les dimensions beaucoup plus réduites, le vase de « La Caillouerie " est très similaire à celui découvert sur le site de l'alignement du moulin de Cojou à Saint-Just (Ille-et-Vilaine; fig. 3). Ce dernier a fait l'objet d'une datation par le radiocarbone à partir d'un charbon présent à l'intérieur. Malgré un écart-type très élevé qui offre une datation recouvrant près de l'ensemble du III ${ }^{e}$ millénaire avant notre ère (GIF $5235: 3940+/-80$ BP, soit 2835-2151 cal BC; Le Roux et al., 1983), ce type de céramique est aujourd'hui considéré par de nombreux auteurs comme étant attribué à l'âge du Bronze ancien et de tradition campaniforme (Gomez de Soto, 1982; Besse, 2003; etc.).

\section{3. ÉTUDE DE L'ASSEMBLAGE LITHIQUE}

\section{Corpus et provenance des lots}

La série lithique étudiée ici, composée de 10072 pièces (6693, hors esquilles), correspond uniquement à la concentration $\mathrm{n}^{\circ} 2$. Ce choix a été conditionné par le fait qu'il s'agissait du seul mobilier issu de l'opération de sauvetage et qu'il se situait en place, protégé par le massif dunaire, et par conséquent peu touché par l'érosion littorale et les piétinements, contrairement à la concentration $\mathrm{n}^{\circ} 1$.

Les tentatives de raccords physiques ont permis de remonter trente-sept galets fractionnés en deux, deux éclats ensemble, ainsi qu'un même éclat brisé en deux. De plus, quatre éclats corticaux ont pu être identifiés comme provenant du même galet, même si l'on n'a pu les raccorder physiquement (texture et teinte de la silicification identiques, cortex semblable, etc.). Cela représente donc un total de 


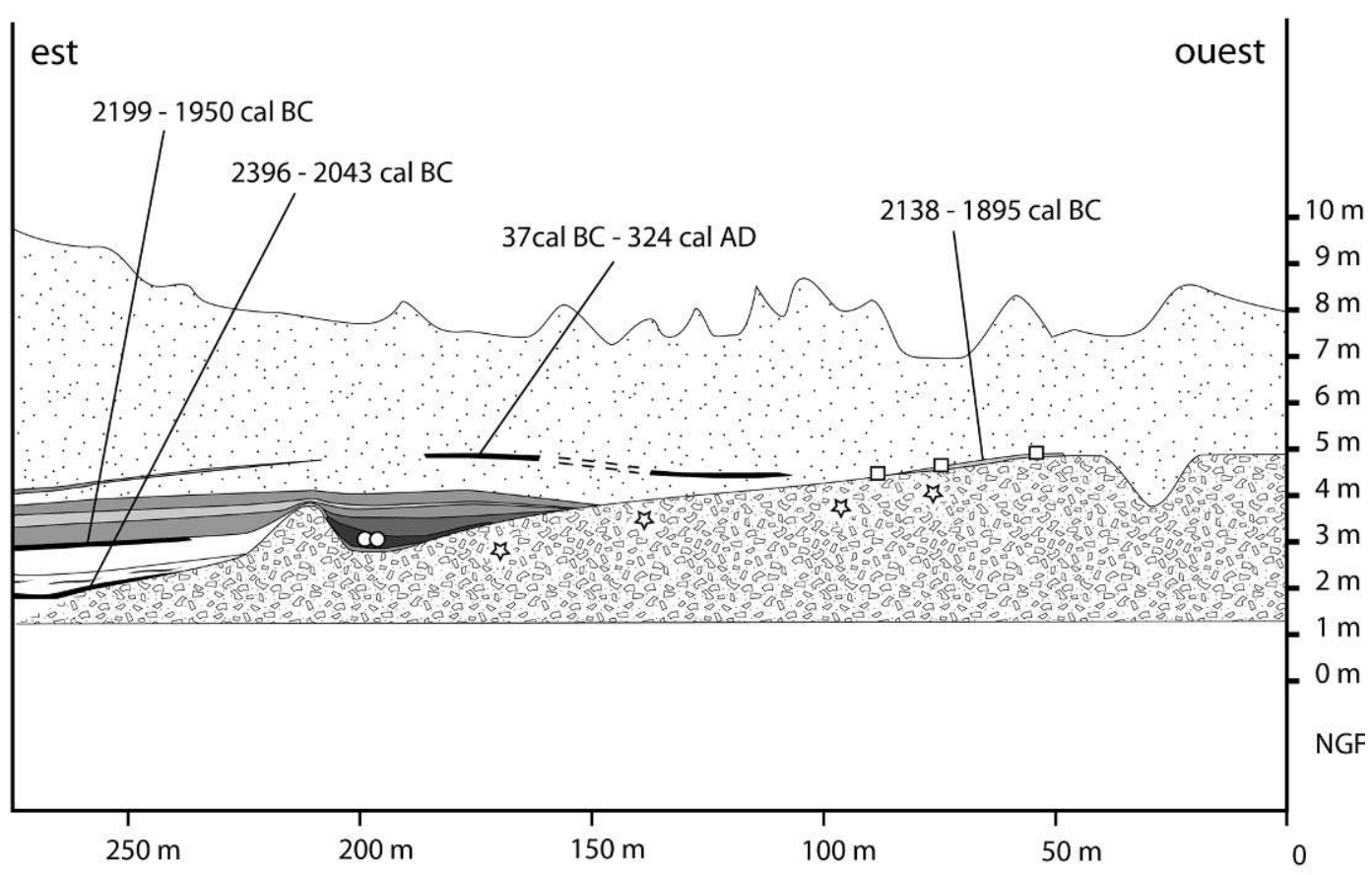

LEGENDE
sable dunaire orangé
tangue sableuse faiblement litée (schorre)
tangue sableuse et lits de sables intercalés
sable argileux
sables fluviatiles fins clairs
silts tourbeux à restes végétaux
échelle des hauteurs = 10X Xéchelle des longueurs

Figure 2 : Saint-Lô d'Ourville "La Caillouerie " : extrait de la coupe stratigraphique du havre de Portbail. D'après Billard et al., 2006; modifié.

Figure 2: Saint-Lô d'Ourville "La Caillouerie": extract from the stratigraphical cut of Le havre de Port-Bail. From Billard et al., 2006, modified.

\begin{tabular}{|c|c|c|c|c|c|c|}
\hline $\begin{array}{l}\text { Code } \\
\text { laboratoire }\end{array}$ & Prélèvement & Contexte & Datation BP & $\begin{array}{c}\text { Datation calibrée } \\
\text { (d'après Billard et al. } \\
\text { 2006) }\end{array}$ & $\begin{array}{c}\text { Datation calibrée } \\
\text { (d'après le logiciel } \\
\text { OxCal, v. } 4.2 \text { ) }\end{array}$ & $\begin{array}{c}\text { Pic de probabilité } \\
\text { (d'après le logiciel } \\
\text { OxCal, v. } 4.2 \text { ) }\end{array}$ \\
\hline Ly-13901 & Tourbe & $\begin{array}{c}\text { Niveau tourbeux } \\
\text { (fond de marécage palustre) }\end{array}$ & $3785+/-40$ & $2396 / 2043 \mathrm{cal} \mathrm{BC}$ & $2397 / 2043 \mathrm{cal} \mathrm{BC}$ & $2346 / 2124 \mathrm{cal} \mathrm{BC}$ \\
\hline Ly-13090 & $\begin{array}{l}\text { Sédiment } \\
\text { organique }\end{array}$ & $\begin{array}{c}\text { Niveau organique (fond de marécage } \\
\text { palustre à saumâtre) }\end{array}$ & $3690+/-40$ & $2199 / 1950 \mathrm{cal} \mathrm{BC}$ & $2199 / 1960 \mathrm{cal} \mathrm{BC}$ & $2152 / 1960 \mathrm{cal} \mathrm{BC}$ \\
\hline Ly-2109 & Os & Niveau archéologique & $3650+/-40$ & $2138 / 1895 \mathrm{cal} \mathrm{BC}$ & $2140 / 1914 \mathrm{cal} \mathrm{BC}$ & $2140 / 1914 \mathrm{cal} \mathrm{BC}$ \\
\hline UL-2320 & Tourbe & Lentille silto-organique & $1870+/-70$ & - & $37 \mathrm{cal} \mathrm{BC} / 324 \mathrm{cal} \mathrm{AD}$ & $21 \mathrm{cal} \mathrm{BC} / 263 \mathrm{cal} \mathrm{AD}$ \\
\hline
\end{tabular}

Tableau 1 : Saint-Lô d'Ourville " La Caillouerie » : datations du site par le radiocarbone. D'après Billard et al., 2006.

Table 1: Saint-Lô d'Ourville "La Caillouerie": site radiocarbon dating. From Billard et al., 2006. 

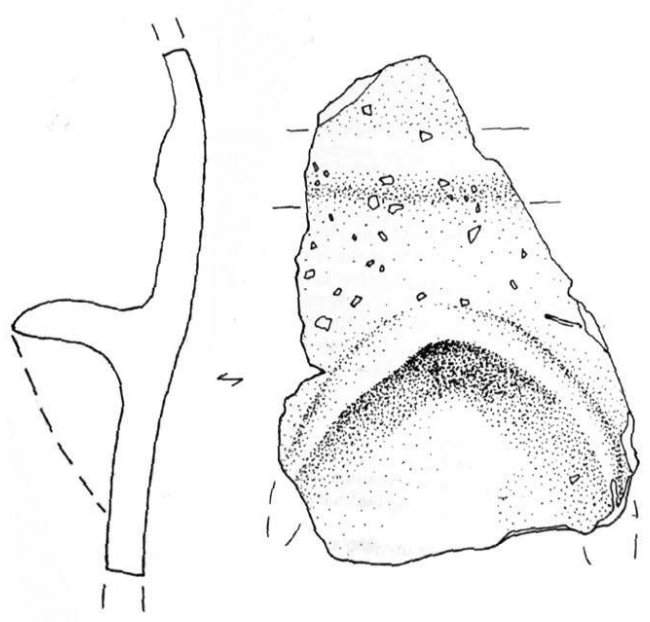

0 $5 \mathrm{~cm}$

\section{Saint-Lô d'Ourville «La Caillouerie» (50) (d'après Billard et al., 2006)}

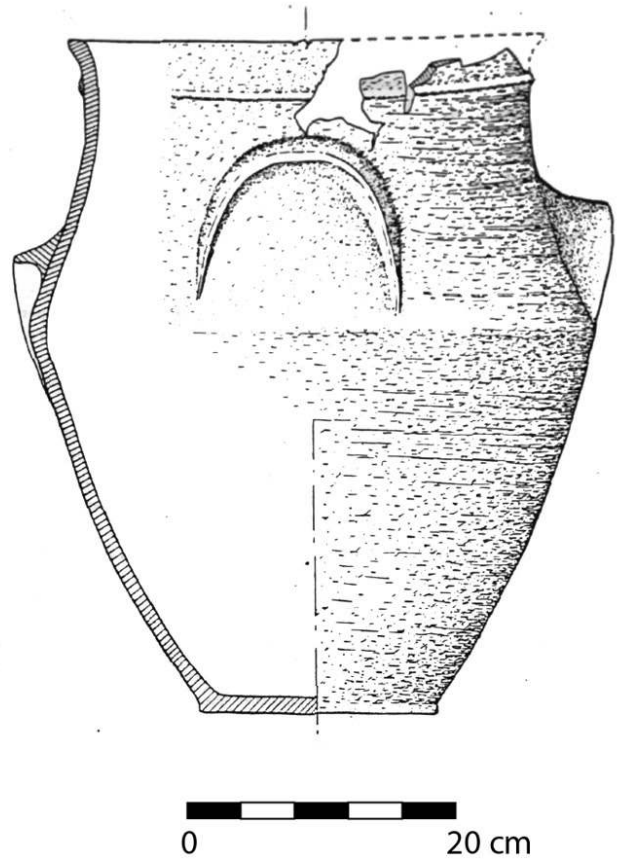

Saint-Just «Alignement du Moulin de Cojou» (35) (d'après Le Roux et al., 1983)

Figure 3 : Saint-Lô d'Ourville "La Caillouerie » : élément comparatif du tesson à anse arciforme et cordon préoral. Figure 3: Saint-Lô d'Ourville "La Caillouerie": comparative element of sherd with horse shoe handle and preoral cordon.

84 pièces remontées sur un total de 6485 éléments de débitage (hors esquilles), soit $1,3 \%$ du lot.

Il est important de noter que lors de l'enregistrement des données en laboratoire, les macro-outils et les blocs brisés en plusieurs éléments ont été décomptés comme un seul individu malgré leur fractionnement. Par conséquent, les raccords physiques concernent quatre individus :

- seize fragments d'un bloc de roche gris foncé de lithologie indéterminée ont été identifiés, dont les trois plus gros morceaux remontent assurément. Ce bloc semble avoir été brisé intentionnellement dans le sens longitudinal;

- un galet de grandes dimensions (environ $11 \mathrm{~cm}$ par 10) ayant servi d'enclume et de percuteur, a pu être remonté partiellement (cinq fragments retrouvés);

- une autre enclume, aménagée sur un bloc de grès-quartzite brisé en onze morceaux, peut être reconstituée;

- enfin, une dernière enclume en roche sédimentaire (calcaire?) est cassée en quatre morceaux.

\section{Les matières premières utilisées}

Les matières premières utilisées proviennent quasi exclusivement de petits galets de silex (99\%), plus rarement de quartz, de grès, de quartzite ou de granite, mesurant entre 3 et $7 \mathrm{~cm}$ de long. Le silex est majoritairement blanc à gris opaque, légèrement patiné, de qualité clastique souvent médiocre, avec un cortex très roulé plus ou moins fin, voire absent (fig. 4). Concernant le macro-outillage, l'utilisation de roches sédimentaires siliceuses (grès, quartzite, etc.), sous la forme de blocs décimétriques au cortex roulé attestant un charriage marin ou fluviatile, est systématique (tableau 2). L'ensemble de ces matériaux provient du cordon littoral sous-jacent au niveau d'occupation et qui affleure en pied de plage.

Les stigmates d'une altération thermique par le feu ne sont quasiment pas attestés sur le site. Seuls trois éclats en possèdent, ce qui est anecdotique à l'échelle de la station et ne perturbe en rien l'identification des matériaux. 

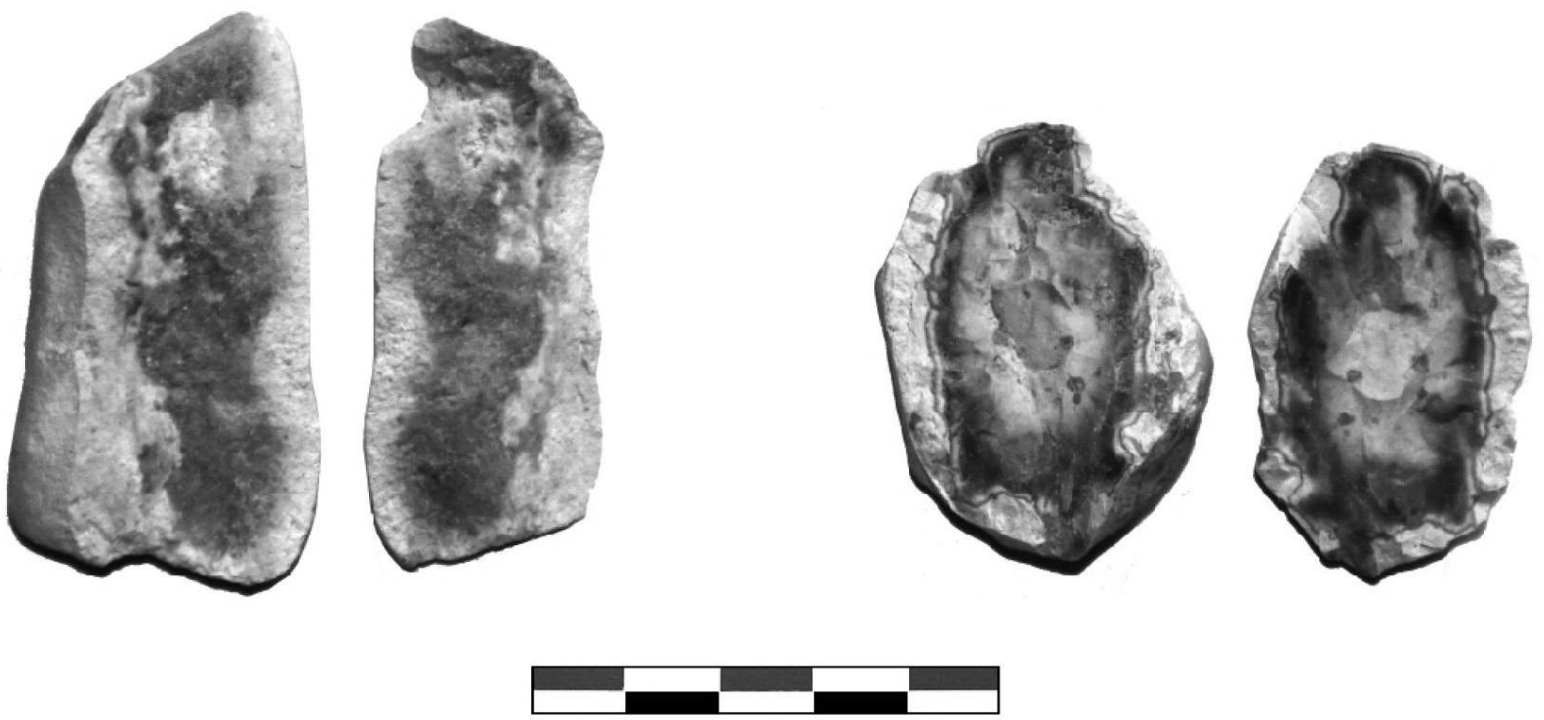

Figure 4 : Saint-Lô d'Ourville "La Caillouerie " : galets côtiers de silex fendus en deux. Cliché L. Rousseau. Figure 4: Saint-Lô d'Ourville "La Caillouerie": coastal flint pebbles split in two. Photo L. Rousseau.

\begin{tabular}{|l|c|c|c|c|c|c|c|}
\hline & & & & & \\
Typologie & Silex & Quartz & Grès & Quartzite & Granite & Indéterminé & Totaux \\
\hline Galet & 75 & 28 & 11 & 3 & 2 & 4 & $\mathbf{1 2 3}$ \\
\hline Test & 215 & 5 & - & 3 & - & - & $\mathbf{2 2 3}$ \\
Nucléus & 454 & - & - & - & - & - & $\mathbf{4 5 4}$ \\
\hline Eclat cortical & 3316 & 3 & 8 & 3 & - & - & $\mathbf{3 3 3 0}$ \\
Eclat à réserve corticale & 1739 & - & - & - & - & - & $\mathbf{1 7 3 9}$ \\
Eclat de plein débitage & 727 & 1 & - & - & - & - & $\mathbf{7 2 8}$ \\
\hline Laminaire & 5 & - & - & - & - & - & $\mathbf{5}$ \\
\hline Esquille & 3379 & - & - & - & - & - & $\mathbf{3 3 7 9}$ \\
\hline «Pièce esquillée $»$ & 69 & - & - & - & - & - & $\mathbf{6 9}$ \\
\hline Eclat utilisé & 6 & - & - & - & - & - & $\mathbf{6}$ \\
Lamelle utilisée & 1 & - & - & - & - & - & $\mathbf{1}$ \\
\hline Grattoir & 6 & - & - & - & - & - & $\mathbf{6}$ \\
\hline Enclume & - & - & 2 & - & - & 2 & $\mathbf{4}$ \\
Percuteur-enclume & 1 & - & 1 & - & - & - & $\mathbf{2}$ \\
Maillet & - & - & 1 & - & - & - & $\mathbf{1}$ \\
Pilon & - & - & - & - & - & 1 & $\mathbf{1}$ \\
Galet biseauté & - & - & - & - & - & 1 & $\mathbf{1}$ \\
\hline Totaux & 9993 & $\mathbf{3 7}$ & $\mathbf{2 3}$ & $\mathbf{9}$ & $\mathbf{2}$ & $\mathbf{8}$ & $\mathbf{1 0 0 7 2}$ \\
\hline Pourcentage & $99 \%$ & Infime & Infime & Infime & Infime & Infime & $\mathbf{1 0 0 \%}$ \\
\hline Dont altération thermique & 3 & - & - & - & - & - & $\mathbf{3}$ \\
\hline
\end{tabular}

Tableau 2: Saint-Lô d'Ourville " La Caillouerie " : composition typologique du mobilier par matières premières.

Table 2: Saint-Lô d'Ourville "La Caillouerie": typological composition of the artifacts according to raw materials.

\section{Le débitage}

La présence de l'ensemble des éléments de la chaîne opératoire (fig. 5) - à savoir la fréquence élevée d'éclats en tous genres (corticaux ou non), d'esquilles, de nucléus, de galets bruts et testés, ainsi que d'outils et de macro-outils - suggère la réalisation d'un débitage effectué in situ.

\section{Les nucléus}

L'abandon de nombreux galets testés ne présentant qu'un ou deux enlèvements (223 individus) témoigne de leur mauvaise qualité (galets fissurés ou gélifractés).

Les nucléus, également très nombreux, totalisent 454 individus issus de petits galets côtiers de silex débités et présentent des enlèvements bipolaires (69\%), unipolaires (27\%), voire orthogonaux (4\%). La percussion posée sur enclume, engagée ici, se caractérise notamment par la présence de stig- 


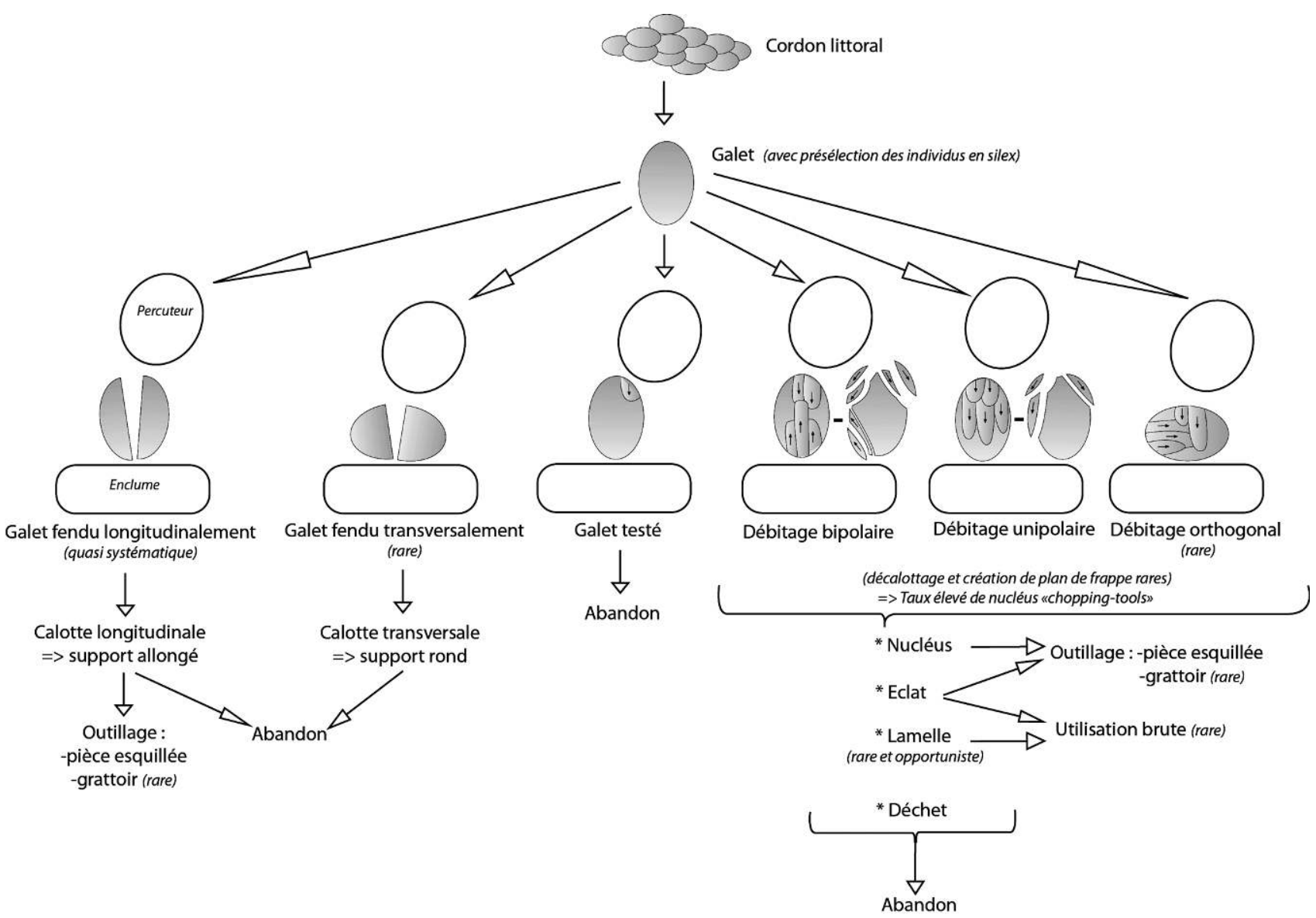

Figure 5 : Saint-Lô d'Ourville « La Caillouerie " : schéma de la chaîne opératoire du site. DAO L. Rousseau.

Figure 5: Saint-Lô d'Ourville "La Caillouerie": pattern of the chaîne opératoire of the site. CAD L. Rousseau.

mates laissés, par le contrecoup de la percussion, au niveau du point de contact entre le galet et l'enclume (écrasements plus ou moins marqués et/ou enlèvements de matière). Les galets, de par leur convexité naturelle, n'ont fait l'objet d'aucune préparation ni d'aucune mise en forme. L'ouverture d'un plan de frappe n'a été que rarement identifiée, ce qui confère à la majorité des nucléus une morphologie dite en "chopping-tools" (Joussaume, 1981; Guyodo et Marchand, 2005 ; fig. 5). La présence de très larges plages corticales sur presque tous les nucléus démontre une très courte séquence d'exploitation des galets et un abandon rapide.

Enfin, quatre nucléus se distinguent du lot. Dépourvus de cortex, leur débitage s'exprime par percussion directe dure, à partir de plans de frappe uniques $(2 \mathrm{cas})$, voire multiples (2 cas). Deux d'entre eux témoignent d'une exploitation optimale du nucléus si l'on se réfère à leur extrême petitesse $(\leq 2 \mathrm{~cm})$.

\section{Les produits de débitage}

Le débitage, impliquant une chaîne opératoire simple et tributaire du matériau employé (galets de silex), est essen- tiellement tourné vers une production d'éclats courts non standardisés et de calottes corticales réalisées par le bris des galets en deux éléments similaires.

Les éclats totalisent ainsi 5797 éléments (cassons compris). Ils sont le plus souvent corticaux $(57,5 \%$, dont $27 \%$ de calottes très majoritairement longitudinales) ou à réserves corticales (30\%). Les éclats dépourvus de cortex sont présents, mais en faible proportion (12,5\%), ce qui indique de nouveau une chaine opératoire courte et un abandon rapide des nucléus.

Les éclats sont essentiellement tirés par percussion posée sur enclume, si l'on se réfere à leur morphologie (talon souvent écrasé, partie distale parfois esquillée, face inférieure vibrée et absence de bulbe), ainsi qu'au taux élevé d'accidents Siret (engendrant de nombreux éclats en "bâtonnet ", " frite » et " quartier d'orange »). La percussion directe à la pierre dure est également attestée dans toutes les phases de débitage, mais dans une moindre mesure (fig. 6). Il faut cependant rester prudent avec ces observations. En effet, R. Furestier a récemment signalé la difficulté de faire la différence entre des éclats extraits par percussion directe dure lancée " classique » et les éclats extraits par percussion «inté- 

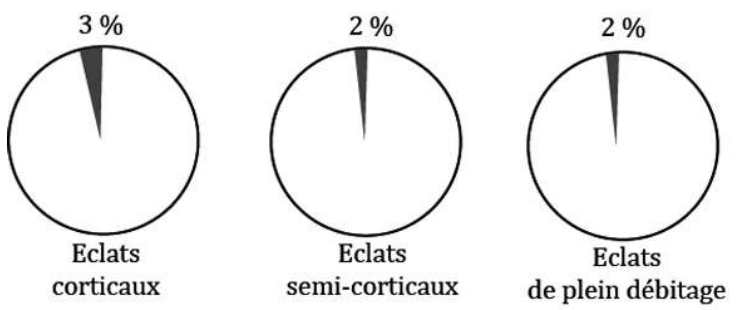

Enclume

Percussion directe dure
Figure 6 : Saint-Lô d'Ourville "La Caillouerie ": techniques de débitage employées dans la production d'éclats.

Figure 6: Saint-Lô d'Ourville "La Caillouerie": Alaking technique used in flakes production. rieure oblique " posée sur enclume (percussion rentrante avec "désaxement $[. .$.$] entre le point de percussion et le$ point d'ancrage sur l'enclume»), car tous deux peuvent posséder les mêmes caractéristiques, à savoir des talons lisses et larges, sans écrasements distaux (Furestier, 2011; p. 193).

Les supports laminaires sont vraiment anecdotiques : trois lamelles à deux pans dépourvues de cortex et deux à trois pans. Aucune volonté spécifique dans l'obtention de ce type de support n'est à signaler, car il s'intègre pleinement dans les séquences de productions d'éclats.

Enfin, les esquilles $(\leq 1 \mathrm{~cm})$ sont fortement représentées (3 379 individus). Ce facteur est à mettre en lien avec le fait que les terres issues de l'intervention archéologique ont été tamisées et que les esquilles étaient particulièrement bien visibles sur la plage de sable.

\section{Le cas des "pièces esquillées", apport de l'analyse tracéologique}

La pièce esquillée - " outil ou déchet? " (Mazière, 1984) - est sujette à débat depuis sa mise en évidence, il y a déjà plus d'un siècle (Bardon et al., 1906). En effet, la distinction entre l'outil ayant servi de pièce intermédiaire et le nucléus débité par percussion bipolaire sur enclume n'est pas toujours aisée, car ils possèdent tous les deux des critères morphologiques proches. De plus, la caractérisation de la " pièce esquillée " se veut plus ou moins stricte selon les auteurs :

- certains considèrent qu'elle ne peut être réalisée qu'à partir d'un support assez fin dépourvu de cortex et qu'elle se caractérise par " un esquillement marqué et continu sur tout le pourtour de la pièce " (Hayden, 1980; Donnart et al., 2009);

- d'autres intègrent tous types de supports (du petit bloc au fragment d'éclat, en passant par le nucléus) et signalent une forme généralement quadrangulaire de l'outil qui peut posséder une à quatre parties actives (Guyodo et Marchand, 2005; Le Brun-Ricalens, 2006).

De plus, des expérimentations ont montré que les stigmates de la percussion bipolaire posée sur enclume et ceux des supports utilisés comme pièces intermédiaires étaient souvent très similaires, ce qui rendait la distinction quasiment impossible (Peña Alonso, 2011). Il est cependant signalé que lors d'une utilisation comme coin pour couper des os longs, la distinction pouvait se faire au niveau des parties actives esquillées. En effet, sur la pièce esquillée-outil, la partie percutée possède une délinéation rectiligne tandis que la partie en contact avec l'os est souvent irrégulière; alors que sur le nucléus, elles sont toutes les deux symétriques (ibid.).

C'est ainsi que, dans un premier temps, ont été considérés comme pièces esquillées tous les supports (nucléiformes et de type éclat) comportant clairement une ou plusieurs parties actives prenant la forme de "tranchants " linéaires, fins et très esquillés. À ce stade, il était fort probable que certaines pièces soient des nucléus bipolaires très largement percutés. C'est pourquoi, une étude tracéologique a été réalisée dans le cadre de ce travail ${ }^{2}$.

Un total de 69 pièces a été isolé (fig. 7, $\mathrm{n}^{\text {os }} 1-7,10,13$ ), toutes issues de galets côtiers en silex. Les supports concernés sont soit nucléiformes, soit des éclats assez épais plus ou moins corticaux, et ils témoignent tous d'un débitage par percussion posée sur enclume. Ces pièces, dont les dimensions sont conditionnées par la forme du support d'origine, mesurent 25-49 mm de long, pour 20-49 $\mathrm{mm}$ de large et 8-23 mm d'épaisseur. Les parties actives sont le plus souvent doubles et opposées, parfois simples et plus rarement triples, voire quadruples, avec des esquillements très prononcés, signes d'une utilisation ou percussion assez violente. Elles sont à plus de $80 \%$ rectilignes, dans de rares cas concaves ou convexes et mesurent en moyenne $22 \mathrm{~mm}$ de long.

L'ensemble des artefacts a fait l'objet d'une expertise tracéologique pour tenter de déterminer si elles pouvaient être uniquement considérées comme des déchets techniques issus du débitage par percussion posée sur enclume, ou si certaines avaient connu une utilisation fonctionnelle.

Dans l'ensemble, la série présente un mauvais état de surface, du fait de diverses altérations chimiques (patine) ou mécaniques qui ne permettent pas une observation à fort grossissement. L'expertise tracéologique repose donc sur l'examen des traces visibles à faible grossissement $(10 \mathrm{x}$ à $48 \mathrm{x}$ ) à la loupe binoculaire (émoussés, stries et écaillures).

La totalité des objets observés porte les traces caractéristiques d'une action en percussion indirecte, la pièce étant l'intermédiaire entre une surface percutée et un percuteur.

2. Étude tracéologique réalisée par François Charraud. 


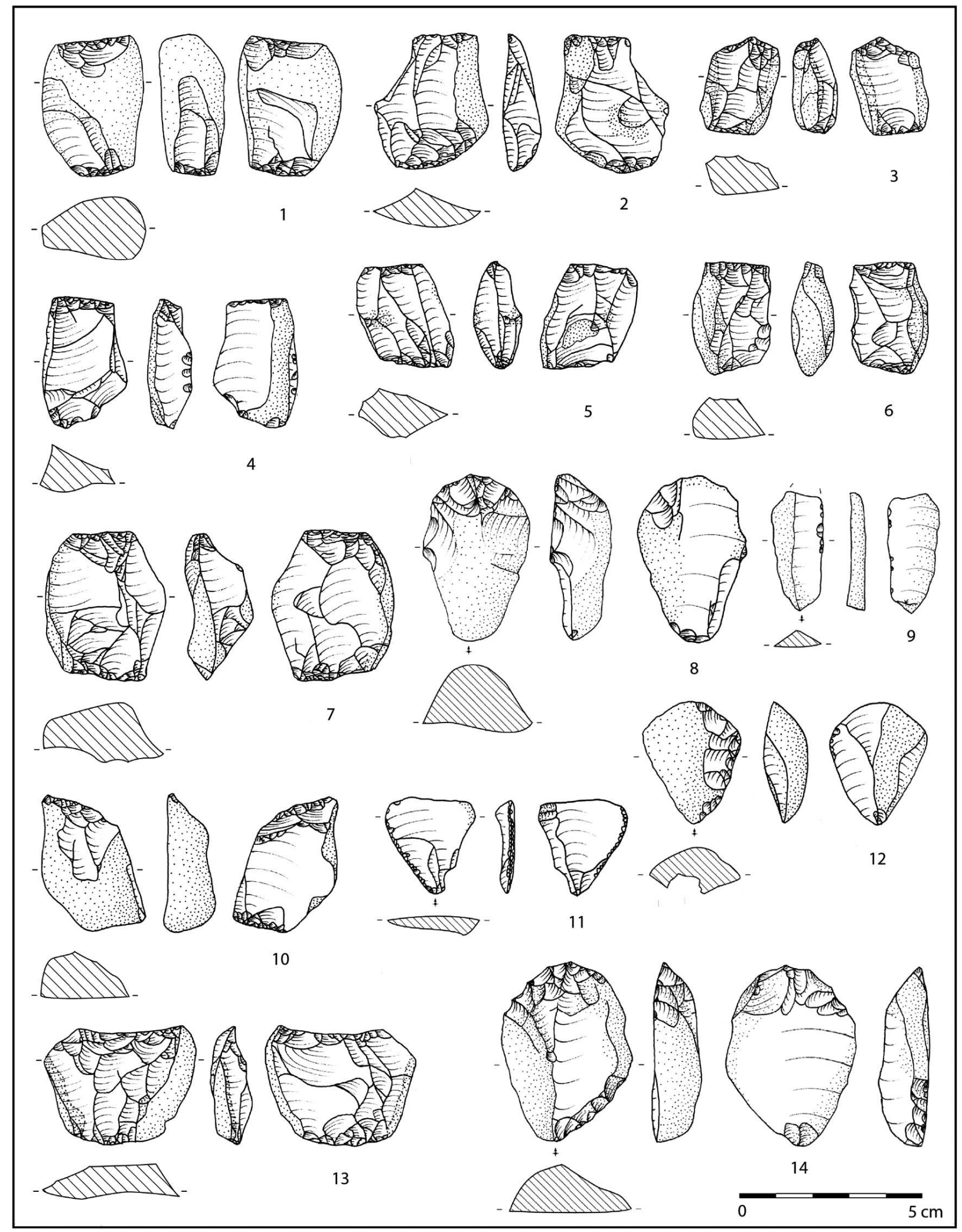

$1-7,10,13$ : pièce esquillée ; 8,12 : grattoir simple ; 9 : lamelle utilisée ; 11 : éclat utilisé ; 14 : grattoir double

Figure 7 : Saint-Lô d'Ourville "La Caillouerie " : mobilier lithique. Dessins : L. Rousseau.

Figure 7: Saint-Lô d'Ourville "La Caillouerie": stone artefacts. Drawings : L. Rousseau. 
Cette action est signalée quasi systématiquement par deux groupes de traces opposés sur les bords de la pièce : sur un bord se trouvent des traces provoquées par l'impact du percuteur; sur le bord opposé figurent les traces formées par contrecoup au contact de la matière d'œuvre ou de l'enclume (là est toute la question).

Pour l'essentiel des individus (64 sur 69), ces traces signalent un contact avec une matière minérale dure : quelques écaillures, courtes et à terminaison réfléchie, sont rapidement altérées par une fissuration importante de la zone percutée, ou son écrasement en cas d'impacts répétés. Aucun émoussé ne se développe du fait de la dureté de la matière d'œuvre et de la rapidité de l'altération. Ces traces sont vraisemblablement d'origine technologique.

De rares pièces (5) présentent toutefois les stigmates d'une action sur matière organique dure : les écaillures sont plus longues, à terminaison réfléchie ou infléchie, aux surfaces d'éclatement vibrées. Les parties saillantes du bord actif sont adoucies par un émoussé arrondi luisant, incompatible avec un contact sur minéral dur. Ces traces sont toujours à l'opposé du bord percuté, ce qui suggère un usage comme coin à fendre. Une pièce présente une partie active concave en segment de cercle, évoquant le travail d'un objet cylindrique de petit diamètre (15 $\mathrm{mm}$ environ).

En définitive, l'étude fonctionnelle permet de constater que seulement $7 \%$ des artefacts isolés (soit cinq pièces) paraissent bien avoir été employés pour le travail de matières organiques dures.

\section{L’outillage}

Le site de "La Caillouerie » se caractérise par un outillage peu fourni. En effet, en plus des cinq pièces esquillées signalées ci-dessus (dont la fonction d'outil a été avérée), six grattoirs et quelques supports bruts présentant des stigmates d'utilisation, complètent l'ensemble.

Les six grattoirs du site sont tous issus de petits galets côtiers de silex débités par percussion posée sur enclume (fig. $7, \mathrm{n}^{\text {os }} 8,12,14$ ). Les supports employés sont souvent des éclats épais corticaux à semi-corticaux, voire des calottes longitudinales, et un individu a été aménagé sur un support nucléiforme. Les parties actives sont majoritairement uniques (si l'on excepte le grattoir double sur calotte) et sont positionnées de manière aléatoire : deux latéralisées à droite, une à gauche et deux axiales distales. Le grattoir double présente un front semi-circulaire dans l'axe de débitage et un second front en partie proximale, légèrement latéralisé à droite (fig. $7, \mathrm{n}^{\circ} 14$ ). Les retouches, directes et semi-abruptes à abruptes et courtes, montrent une réalisation sommaire et sont parfois effectuées sur des supports de très mauvaise qualité (fig. $7, \mathrm{n}^{\circ} 12$ ), probablement pour un usage ponctuel.
Deux supports présentent des enlèvements bifaciaux, courts et écailleux pour l'un (support nucléiforme) et inverses, courts et écailleux pour le second (éclat). Cinq autres supports possèdent des micro-esquillements situés au niveau des bords ou des extrémités : il s'agit soit d'éclats légèrement corticaux ou non (trois cas; fig. $7, \mathrm{n}^{\circ} 11$ ), d'éclat laminaire (un cas) ou de lamelle semi-corticale à deux pans (un cas; fig. $7, \mathrm{n}^{\circ}$ 9). Cette diversité de supports utilisés ne signale aucune prédétermination dans leur choix.

\section{Le macro-outillage}

\section{Les enclumes}

Les enclumes sont au nombre de six si l'on y ajoute les macro-outils composites cités ci-dessous (fig. 8, $\mathrm{n}^{\text {os }} 4$-7). Les supports utilisés sont systématiquement des blocs roulés de roche sédimentaire siliceuse (grès beige, 2; grès-quartzite beige, 1 ; silex blanc, 1 ; roche indéterminée de teinte grise, calcaire? 2), de formes ovoïdes, parallélépipédiques à subparallélépipédiques. Les dimensions sont variables et ne semblent pas être un critère de choix, car des enclumes de petite taille $(86 \times 59 \mathrm{~mm})$ peuvent côtoyer des plus grandes $(164 \times$ $144 \mathrm{~mm}$ ). Il en va par conséquent de même concernant le poids des blocs qui peut varier de $300 \mathrm{~g}$ à près de $1500 \mathrm{~g}$. Seule la présence d'une surface plane semble être le critère déterminant. Les enclumes possèdent une à quatre plages de percussion formant, dans la plupart des cas, des cupules assez profondes par des enlèvements de matière. Elles sont le plus souvent complètes, mais la violence des coups a brisé l'une d'entre elles en quatre fragments, et a provoqué (sur un second exemplaire) de larges fissures. Enfin, une dernière enclume a pu être partiellement reconstituée à partir de onze fragments de grès. La fragmentation de cette dernière, probablement due à un choc plus qu'à sa fonction d'enclume, a été facilitée par la nature même du matériau employé qui se délite horizontalement.

\section{Les percuteurs-enclumes}

Deux macro-outils composites, ayant servi à la fois de percuteur et d'enclume, ont été identifiés. Le premier est un galet de grès beige de dimensions moyennes $(86 \mathrm{~mm} \mathrm{x}$ $59 \mathrm{~mm} \times 57 \mathrm{~mm}$ ) comportant une plage de percussion sur l'une de ses extrémités. La présence d'une cupule de percussion au centre de l'une des surfaces du bloc indique qu'il a également été utilisé comme enclume, mais de manière très modérée, étant donné la taille réduite de la cupule (moins d'1 $\mathrm{cm}^{2}$; fig. $\left.8, \mathrm{n}^{\circ} 4\right)$.

Le second est un galet de silex blanchâtre et opaque possédant un cortex roulé et piqueté (traces en " coups d'ongles ») lié à un charriage maritime/fluviatile très violent. Ce bloc, mesurant une dizaine de centimètres environ, est brisé en 


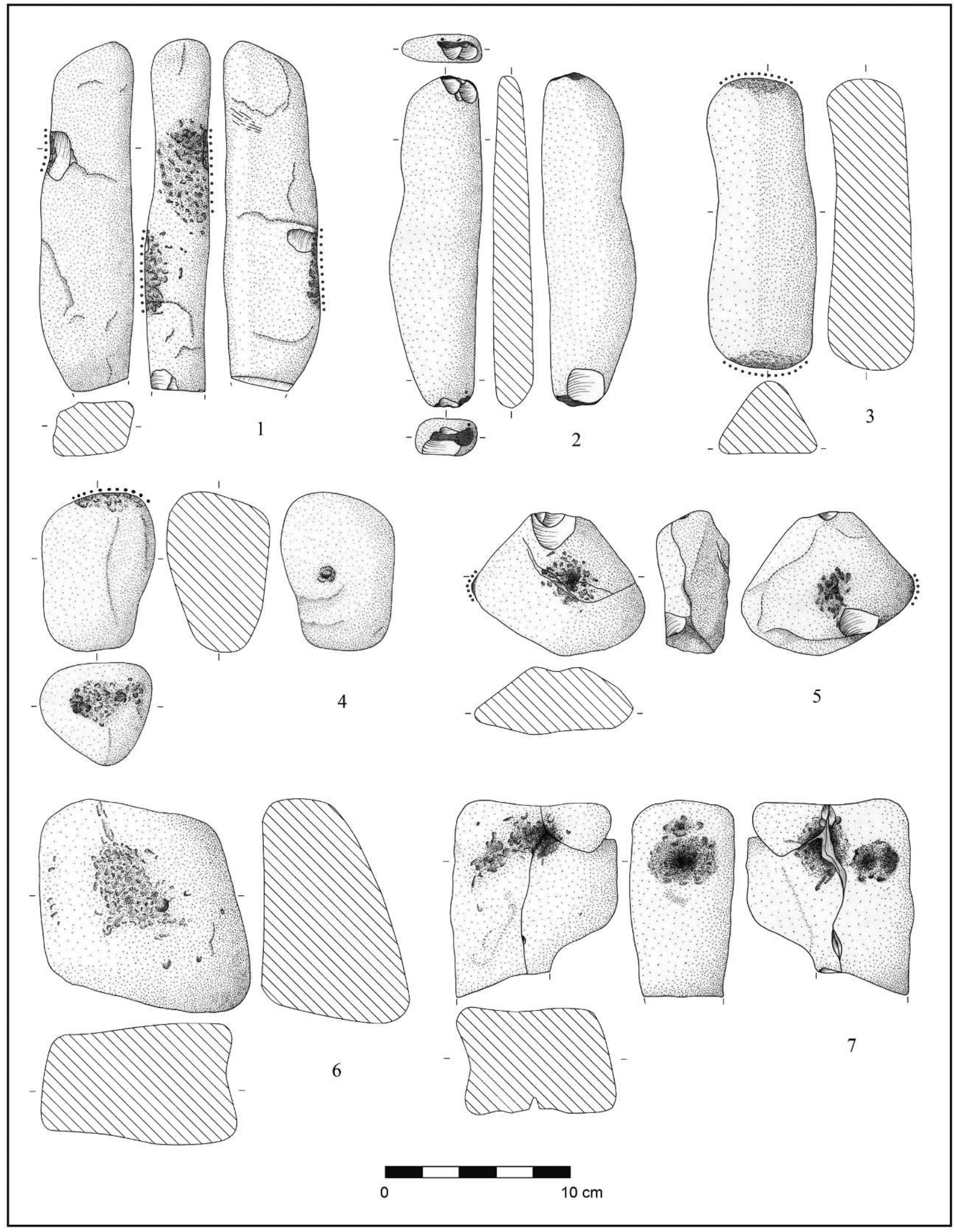

1 : maillet ; 2 : galet biseauté ; 3 : pilon ; 4 : percuteur-enclume ; $5-7$ : enclume

Figure 8 : Saint-Lô d'Ourville " La Caillouerie " : macro-outillage. Dessins : L. Rousseau.

Figure 8: Saint-Lô d'Ourville "La Caillouerie": ground stone tools. Drawings : L. Rousseau. 
plusieurs morceaux dont cinq ont pu être retrouvés (sur 7 ou 8 probables). Deux cupules assez proches sont identifiables sur l'une de ses surfaces : l'une mesure 18 sur $14 \mathrm{~mm}$ et l'autre 13 sur $12 \mathrm{~mm}$. Une des extrémités du galet a servi comme percuteur, ce qui semble avoir contribué à la fracturation de ce dernier, probablement déjà fissuré à l'origine.

\section{Le maillet}

Cet outil est issu d'un bloc roulé parallélépipédique allongé $(190 \times 51 \times 29 \mathrm{~mm})$ de 560 g en grès-quartzite (fig. $8, \mathrm{n}^{\circ} 1$ ). Il possède deux plages de percussion punctiformes, formant dans un cas une cupule, semblables à ce que l'on peut voir sur les autres enclumes. Cependant, leurs positions sur les arêtes du bloc - alors que les faces offraient des surfaces plus étendues et planes pour faire office d'élément passif - suggèrent que cet outil est plutôt un maillet, à savoir un "support allongé pris en main par une extrémité pour frapper sur une pièce intermédiaire avec l'autre » (Donnart, 2007, p. 4).

\section{Le pilon}

Un macro-outil a été confectionné à partir d'un galet allongé à section subtriangulaire ( 156 × 56 x 40 mm, 480 g), caractéristiques qui permettent une bonne prise en main (fig. 8, n $\mathrm{n}^{\circ}$ ). Deux parties actives sont identifiées par des stigmates d'une percussion lancée diffuse aux deux extrémités opposées du support. Il semblerait que cet outil ait été utilisé comme pilon si l'on se réfere à la définition qu'en donne K. Donnart (2007, p. 5) : «C'est un outil rarement mis en évidence dans les séries archéologiques, alors qu'il est attesté ethnographiquement [qui se caractérise] par un support lourd et allongé présentant des traces fines d'écrasement à une ou deux extrémités».

\section{Le galet biseauté}

L'unique galet biseauté du site se présente sous la forme d'un galet allongé et plat $(177 \times 48 \times 21 \mathrm{~mm})$, en roche sédimentaire de couleur gris foncé, de 294 g (fig. 8, n 2). Deux parties actives sont identifiables au niveau des extrémités de la pièce : elles forment un biseau réalisé par des enlèvements (1 à 4) résultant de l'utilisation de l'outil par percussion lancée, auxquels s'ajoutent un écrasement des arrêtes et de légères stries verticales.

\section{Analyse teChNo-fonCtionNelle}

\section{Caractérisation de la production lithique}

L'étude de la série lithique du site de " La Caillouerie " montre un approvisionnement en matières premières exclusivement local. Les macro-outils sont aménagés sur des blocs roulés de roches sédimentaires siliceuses, et le débitage porte sur des petits galets roulés pluri-centimétriques $(3-7 \mathrm{~cm})$, majoritairement en silex, récoltés au sein du cordon littoral sous-jacent au niveau archéologique. Cette profusion de matériaux de proximité et leur qualité souvent très médiocre (gélifractés, fissurés par les chocs marins et de taille réduite) permet d'expliquer l'importante quantité de galets testés et de nucléus ne présentant que quelques négatifs d'enlèvements. Il semble cependant y avoir un choix plus soigné pour les supports des macro-outils : ils sont sélectionnés selon leur future fonction, à savoir un galet plat et allongé pour le galet biseauté, des blocs stables à surface plane pour accueillir les enclumes, un support allongé et adapté à la prise en main pour le maillet, etc. Cette observation a déjà été signalée par K. Donnart concernant d'autres sites de l'âge du Bronze ancien (Donnart, 2012).

Lactivité de débitage par percussion posée sur enclume a produit une quantité importante de calottes longitudinales (rarement transversales), par la séparation d'un galet en deux supports identiques (type I de Joussaume, 1981), d'éclats courts et de supports nucléiformes. La majorité des nucléus atteste un débitage longitudinal simple à enlèvements croisés, créant des nucléus "chopping-tools " (type VI de Joussaume, 1981). Les chaînes opératoires sont complètes, mais elles impliquent des séquences simples et courtes.

Réalisée sur une petite surface, en un temps probablement court, cette " activité " très consommatrice en matière première a engendré une quantité importante de mobilier lithique au sein d'une zone restreinte : environ 10000 artefacts (esquilles prises en compte) sur une surface d'une dizaine de mètres carrés. L'utilisation répétée des enclumes a provoqué de profondes cupules, allant parfois jusqu'à des fragmentations simples à multiples.

Le taux élevé de remontage intercalottes indique qu'une importante quantité d'entre elles ont été délaissées, alors que le silex n'était pas systématiquement de mauvaise qualité. Plusieurs hypothèses peuvent être proposées :

- soit le rejet est lié à la morphologie même des supports obtenus qui sont alors considérés comme trop fins ou trop épais (Guyodo et Marchand, 2005);

- soit la sélection d'une partie des supports a été effectuée au sein de l'amas, suite au débitage successif d'une grande quantité de galets, dans le but d'être transportés hors du gisement, de manière retouchée ou non;

Enfin, l'outillage est quantitativement infime et très peu varié puisqu'il ne correspond à peine plus de $0,25 \%$ des artefacts (hors esquilles) et n'est représenté qu'à travers trois classes d'outils : des grattoirs, des pièces esquillées et des supports vraisemblablement utilisés bruts). Les quelques outils, frustes, ne témoignent par ailleurs d'aucune volonté de standardisation. 


\section{Hypothèses fonctionnelles de l'assemblage lithique}

Après de longs débats sur le statut des pièces esquillées, une utilisation comme outil est aujourd'hui admise, bien que la ressemblance avec certains nucléus complique toujours leur identification au sein des corpus... Malgré tout, plusieurs hypothèses, quant à leur probable fonction, ont été proposées :

- pièces intermédiaires pour décrocher les patelles des rochers situés sur l'estran (Rozoy, 1978; Joussaume, 1981);

- pièces dont les tranchants seraient utilisés pour couper (Mazière, 1984);

- tranchants utilisés afin de racler les hampes d'armes de trait (Guyodo et Marchand, 2005);

- ou encore, coins à fendre ou à fracturer le bois ou les os longs (Mazière, 1984; Dewez, 1985; Lucas et Hays, 2004).

La première hypothèse ne semble pas s'adapter au cas de "La Caillouerie » : pourquoi les pièces esquillées seraientelles restées sur le poste de débitage et non pas sur le lieu de récolte? Si l'on envisage qu'elles ont été rapportées suite à l'activité de ramassage des coquillages, où se trouve la malacofaune associée? (Marchand et Guyodo, 2005).

La dernière hypothèse peut, au contraire, être envisagée puisque l'étude tracéologique effectuée dans le cadre de cette étude a permis de faire un lien possible entre les pièces esquillées du site et des éléments osseux présentant des traces de fractionnements intentionnels récoltés dans le même contexte, même si les modalités exactes du travail de ces objets restent à préciser. Certains ossements présentent, en effet, des fractures longitudinales torses, indiquant une

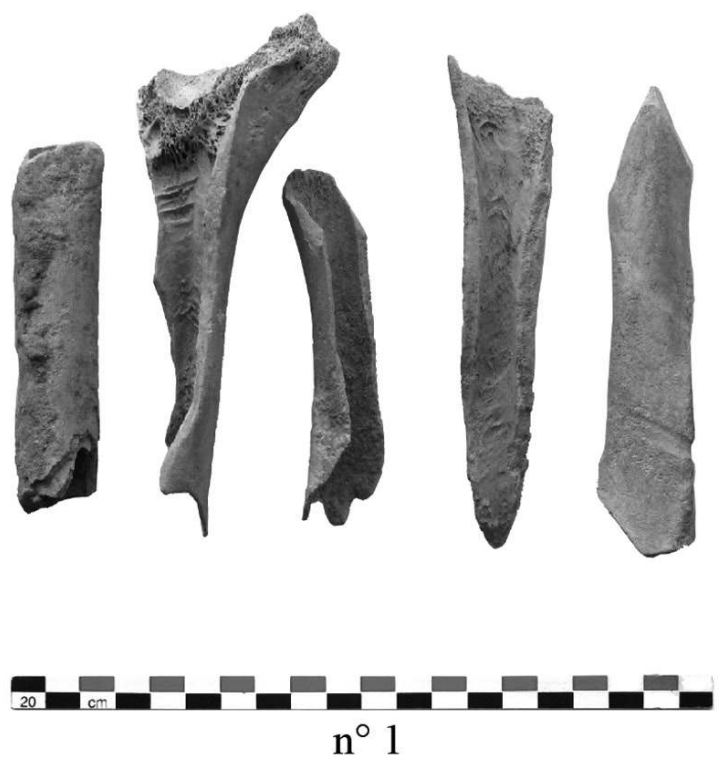

action probablement anthropique sur os plutôt frais (fig. 9, $\mathrm{n}^{\circ} 1$ ). Un des os comporte en outre une rainure oblique en partie proximale et la partie distale a été appointée volontairement (fig. 9, $\mathrm{n}^{\circ}$ 2).

L'hypothèse des "nucléus à esquilles ", concernant les nucléus débités par percussion posée sur enclume, a été rappelée par F. Le Brun-Ricalens (2006), d'après plusieurs éléments ethnographiques. Ces derniers permettent d'obtenir de nombreuses esquilles qui peuvent être fixées sur une lance grâce à une colle naturelle et réaliser ainsi des armes nommées "death-spears", des sortes de harpons (Davidson, 1934). Il s'agit d'une hypothèse intéressante, qui ne pourra malheureusement pas être vérifiée en l'absence de conservation d'une partie des matériaux périssables.

Enfin, l'assemblage macro-lithique correspond quasi exclusivement à l'activité de taille (percuteurs, enclume et maillet). Le pilon laisse penser à une activité ponctuelle de concassage (d'aliments? autres?), au sein du site, sans que l'on puisse en dire davantage. La présence d'un galet biseauté renvoie à l'hypothèse d'un outil utilisé pour décoller les patelles (Pailler et Dupont, 2007), bien que les mêmes considérations que celles effectuées pour les pièces esquillées peuvent être réitérées (cf. supra).

\section{Implications chrono-culturelles et comparaisons}

La stratégie d'acquisition des matières premières implique un faible investissement, tant dans la recherche des matériaux que sur le plan technique : elle est locale et n'engage aucun transport des blocs, dans la mesure où les hommes se sont directement installés sur le cordon pour effectuer leur

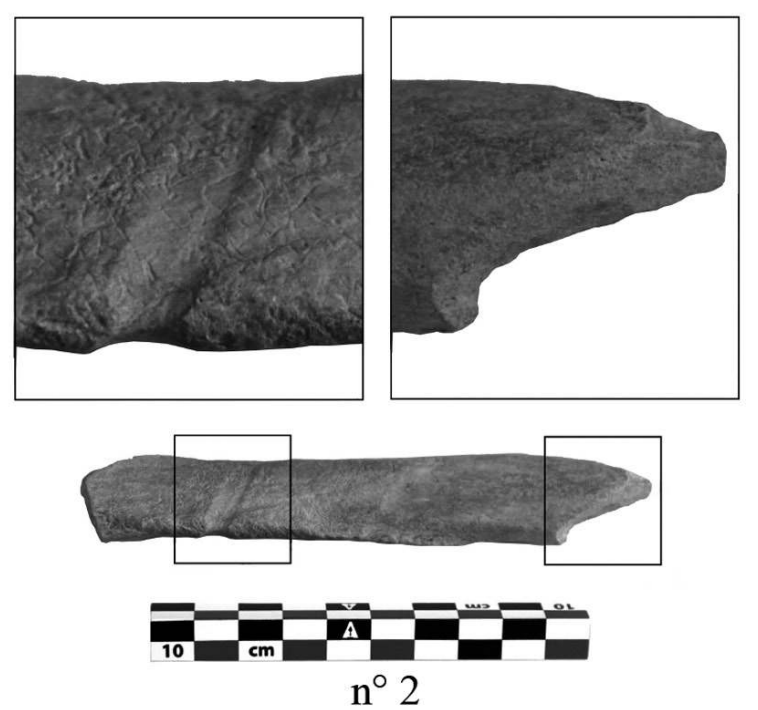

Figure 9 : Saint-Lô d'Ourville "La Caillouerie » : restes fauniques de cervidés. Clichés G. Laisné et L. Rousseau. Figure 9: Saint-Lô d'Ourville "La Caillouerie": cervidae faunal remains. Photos: G. Laisné and L. Rousseau. 
débitage. L'exploitation des ressources minérales locales est un constat généralisé à l'ensemble des sites de la fin du III ${ }^{e}$ et du $\mathrm{II}^{\mathrm{e}}$ millénaire avant notre ère (tableau 3). L'exemple du site de Val-de-Reuil « les Florentins » illustre ce phénomène à l'extrême, car malgré la présence de blocs de silex de bonne qualité à proximité du site, le choix s'est porté sur les médiocres galets situés dans la nappe sous-jacente au niveau d'occupation (Billard et al., 1991). D’une manière générale, la présence de matériaux lithiques exogènes est anecdotique au début de l'âge du Bronze, hormis en contexte funéraire (Nicolas, 2012). Dans la sphère domestique, elle se caractérise le plus généralement sous la forme de rares fragments d'éléments en silex turonien de la région du Grand-Pressigny (exemple : Ancenis " La Savinière 5 "; Viau, 2010), mais aussi par la présence de fragments de lames de haches polies, rarement entières et parfois réemployées (Beauvoir-sur-Mer "Le Pontreau 2 » et Saint-Vaast-la-Hougue « Tatihou » par exemple; Viau, 2006; Marcigny et Ghesquière, 2003). Or, à "La Caillouerie », aucun élément de ce type n'a été découvert.

L'utilisation de la percussion posée sur enclume est couramment attestée au sein des séries lithiques du Campaniforme/Bronze ancien, notamment en contexte littoral (tableau 3). En effet, cette technique se veut totalement adaptée aux petits modules qu'offrent certains galets côtiers qu'il peut être difficile d'ouvrir d'une autre manière. C'est d'ailleurs ce qui avait conduit R. Joussaume (1981) à qualifier cette technique de "débitage côtier ». Or, il convient de préciser qu'elle n'est pas systématiquement engagée sur tous les gisements littoraux et qu'elle a parfois été signalée sur des sites continentaux (Guyodo et Marchand, 2005; tableau 3), mais force est de constater que le module des galets joue un rôle déterminant dans le choix de cette dernière. Bien que souvent identifiée au Bronze ancien, cette technique ne peut cependant être considérée comme ayant une valeur chrono-culturelle (Joussaume, 1981; Marchand et Guyodo, 2005), car elle ne lui est pas spécifique et a été utilisée dès le Paléolithique ancien (Donnart et al., 2009).

La difficulté d'effectuer des comparaisons cohérentes entre sites, d'après la part de chaque type d'outil, réside dans la prise en compte ou non de l'outillage " non-conventionnel $^{3}$ " au sein des études lithiques. En effet, les supports bruts utilisés ne sont pas systématiquement dénombrés, et l'identification des pièces esquillées n'est pas toujours aisée au sein d'un lot où la percussion posée sur enclume est d'usage, car certains nucléus peuvent en avoir quelques caractéristiques :

3. Selon la définition de M. Bailly (2005) : «Pièces lithiques débitées portant des traces d'enlèvements très variés. " "Ce sont des tranchants bruts utilisés. Leur morphologie est donc un état final au moment de l'abandon et non le produit d'une intention de façonnage. " Cela comporte donc « les pièces esquillées, les enlèvement latéraux irréguliers et les enlèvements marginaux ». ce qui explique le fait que certains auteurs préfèreront les considérer systématiquement comme nucléus. Malgré cela, le spectre de l'outillage de "La Caillouerie " s'intègre assez bien à ce que l'on peut observer sur de nombreux sites de la transition $\mathrm{III}^{\mathrm{e}}-\mathrm{II}^{\mathrm{e}}$ millénaire avant notre ère. Lorsque ces outils sont considérés comme tels au sein des séries, ils se retrouvent, avec les grattoirs, dans les trois classes d'outils les plus représentées. Cependant ici, c'est leur faible quantité numérique qui retient l'attention. Peut-être ont-ils été transportés, au même titre que certains supports, sur le site d'habitat probablement en retrait de la plage?

Enfin, la série macro-lithique, essentiellement en cohérence avec l'activité de taille identifiée sur ce site, s'intègre parfaitement au sein des corpus connus (Donnart, 2012). Le pilon et le maillet sont d'ailleurs morphologiquement très proches de ceux découverts sur les sites d'habitats campaniformes de Digulleville «Le Raumarais 2 » (Letterlé et Verron, 1986) et de Saint-Malo " Place des Frères Lamennais" (Hinguant et al., 2007; Donnart, 2012).

\section{ConClusion}

La perduration des productions lithiques au début de l'âge du Bronze commence à être admise, bien que ces dernières semblent a priori de moins en moins abondantes vis à vis des périodes précédentes. En effet, la majorité des sites d'habitat de l'âge du Bronze n'offre que quelques dizaines, voire centaines de pièces (rarement des milliers, seuls « Tatihou »; « le gisement 18 de la Passe de l'Écuissière » et « Beg ar Loued » font exception; Marcigny et Ghesquière, 2003; Laroche, 2012; Pailler et al., 2011), sur des zones décapées parfois assez larges. Comment expliquer cet état de fait? Dans le quart nord-ouest de la France, plusieurs traces de métallurgie ont été identifiées en contexte campaniforme (Val-de-Reuil "Les Florentins », Poses "Vivier-Le Clos Saint-Quentin », Molène "Beg-ar-Loued » et Talmont-Saint-Hilaire "La République 2 "; Dolus-d'Oléron " Passe de l'Écuissière "; Billard et al., 1991; Billard et al., 1994; Pailler et al., 2011; Poissonnier, 1990 ; Querré, 2009). Bien que l'usage du métal ait dû avoir une place importante dès le début de l'âge du Bronze, il est assez difficile d'appréhender son ampleur. En effet, de nombreux sites domestiques de cette époque n'ayant pas ou peu livré de traces d'une métallurgie ni d'objets métalliques (bien que leur absence « archéologique» ne signifie par pour autant qu'il n'y en a pas eu sur le site à un moment donné), nous sommes en droit de nous interroger sur la généralisation du métal. Par conséquent, le phénomène de substitution de la pierre par le métal ne peut être un argument suffisant à lui seul pour expliquer cette " pénurie » lithique. Il est donc légitime de se demander si la faible 


\begin{tabular}{|c|c|c|c|c|c|}
\hline & Site & Gisement & Matières premières & Technique & Bibliographie \\
\hline \multirow{18}{*}{ 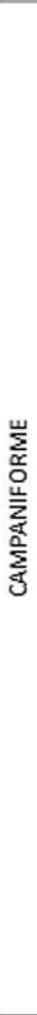 } & Penancreac'h, Quimper (29) & Continental & $\begin{array}{l}\text { Local : grès lustré } \\
\text { Voisin: galets côtiers }\end{array}$ & Enclume & Le Bihan, 1993 \\
\hline & Grand-Rocher, Brétignolles-sur-Mer (85) & Littoral & $\begin{array}{l}\text { Local: galets côtiers } \\
\text { Exogène: } \mathrm{GP} \text { (produit fini) }\end{array}$ & Enclume & Joussaume, 1981 \\
\hline & Marais-Girard, Brétignolles-sur-Mer (85) & Littoral & Local: galets côtiers & Enclume & Joussaume, 1981 \\
\hline & Petit-Rocher, Brétignolles-sur-Mer (85) & Littoral & Local: galets côtiers & Enclume & Joussaume, 1981 \\
\hline & Grouin du Cou, La Tranche-sur-Mer (85) & Littoral & Local: galets côtiers & Enclume & Joussaume, 1981 \\
\hline & Grandes Loges, La Tranche-sur-Mer (85) & Littoral & $\begin{array}{l}\text { Local: galets côtiers } \\
\text { Exogène: silex (mésial de lame) }\end{array}$ & Enclume & Boiral et Joussaume, 1986 \\
\hline & Pointe de Grosse Terre, St-Hilaire-de-Riez (85) & Littoral & Local : galets côtiers & Enclume & Longuet et al., 1985 \\
\hline & Deux-Moulins, Bois-en-Ré (17) & Littoral & Local : galets côtiers & Enclume? & Pautreau et Robert, 1980 \\
\hline & République, Talmont-St-Hilaire (85) & Littoral & Local: galets côtiers & Enclume, PDD & Joussaume, 1981 \\
\hline & Florentins, Val-de-Reuil (27) & Continental & Local: galets de la nappe du sous-sol & Enclume & Billardet al., 1991 \\
\hline & Place Lamennais, St-Malo (35) & Littoral & $\begin{array}{l}\text { Local: galets côtiers } \\
\text { Exogène: quelques éléments }\end{array}$ & Enclume, PDD & Hinguant et al., 2007 \\
\hline & Courance, St-Brévin-les-Pins (44) & Littoral & $\begin{array}{l}\text { Local : galets côtiers } \\
\text { Régional : Moûtiers, Montbert }\end{array}$ & Enclume, PDD & Guyodo, 2001 \\
\hline & Passe de l'Ecuissière, Dolus-d’Oléron (17) & Littoral & Local: galets et blocs & Enclume, PDD & Bougeant, 2009 \\
\hline & Raumarais 2, Digulleville (50) & Littoral & $\begin{array}{l}\text { Local: galets et blocs } \\
\text { Exogène: GP }\end{array}$ & Enclume et PDD & Letterlé et Verron, 1986 \\
\hline & Plaine d'Ectot, Ancrétiéville-St-Victor (76) & Continental & $\begin{array}{l}\text { Local: argiles à silex } \\
\text { Exogène: Caen (Hache) }\end{array}$ & PDD & Ghesquiere et al., 1996 \\
\hline & Clos Bâtard, Tourville-la-Rivière (76) & Continental & Local: « rognons » et rares galets & PDD & Chancerel et Masson, 1991 \\
\hline & Midonnière, St-Lambert-du-Lattay (49) & Continental & $\begin{array}{l}\text { Local: silex de la Loire. } \\
\text { Régional: Thouars, Montbert. } \\
\text { Exogène: GP }\end{array}$ & PDD & Guyodo, 2001 \\
\hline & Grande Pigouille, Belluire (17) & Continental & $\begin{array}{l}\text { Local : terrasses alluviales et argiles à silex } \\
\text { Exogène: turonien saintongeais, GP } \\
\text { (produits finis) }\end{array}$ & PDD & Bouchet et al., 1990 \\
\hline \multirow{6}{*}{ 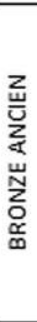 } & Grand Parc, Bernières-sur-Mer (14) & Littoral & Local: galets et blocs roulés & PDD & Marcignyet al., 2003 \\
\hline & Pontreau 2, Beauvoir-sur-Mer (85) & Littoral & $\begin{array}{l}\text { Local : galets côtiers, terrasses anciennes, } \\
\text { Moûtiers }\end{array}$ & Enclume, PDD, PDT & Viau, 2006 \\
\hline & Domaigne, Guilberville (50) & Continental & Local: Caen, galets côtiers & Enclume, PDD & Marcignyet al., 2001 \\
\hline & Savinière 5, Ancenis (44) & Continental & $\begin{array}{l}\text { Local: terrasses alluviales } \\
\text { Exogène: GP, turonien saintongeais, } \\
\text { jurassique sarthois, dolérite } A \text { (produits finis) }\end{array}$ & PDD, PI & Viau, 2010 \\
\hline & Tatihou, St-Vaast-la-Hougue (50) & Littoral & Local : galets côtiers & Enclume, PDD & Marcigny et Ghesquiere, 2003 \\
\hline & Passe de l'Ecuissière 18, Dolus-d'Oléron (17) & Littoral & Local: galets et blocs & Enclume, PDD & Laroche, 2012 \\
\hline \multicolumn{6}{|c|}{ Légende: } \\
\hline \multicolumn{6}{|c|}{$\begin{array}{c}\text { GP (= silex turonien de la région du Grand-Pressigny) } \\
\text { Moûtiers (= silex des Moûtiers-en-Retz) } \\
\text { Montbert (= quartzite de Montbert) } \\
\text { Thouars (= silex de la région du Thouars) } \\
\text { Caen (= silex jurassique de la Plaine de Caen) }\end{array}$} \\
\hline \multicolumn{6}{|c|}{$\begin{array}{l}\text { PDD (=percussion directe pierre dure) } \\
\text { PDT (=percussion directe pierre tendre) } \\
\text { PI (=percussion indirecte) }\end{array}$} \\
\hline & & & Local : $0-5 \mathrm{~km}$; Voisin : $5-30 \mathrm{~km}$ & & \\
\hline
\end{tabular}

Tableau 3: Saint-Lô d'Ourville «La Caillouerie » : localisation, techniques de taille et matières premières employées de quelques sites campaniformes et Bronze ancien du quart nord-ouest de la France.

Table 3: Saint-Lô d'Ourville "La Caillouerie": location, knapping techniques and raw materials used by some Bell Beaker and Early Bronze Age sites in the north-west of France.

quantité de pièces lithiques au sein des noyaux d'habitations n'est pas due au développement d'un système alternatif à la pierre (développement d'outils en matériaux périssables?), ou bien à l'installation des ateliers de débitage directement sur les gisements de matières premières disponibles. Il est ainsi permis d'imaginer que les activités de débitage deviennent de plus en plus ponctuelles à l'âge du Bronze, et qu'elles soient la réponse à un ou plusieurs besoins spécifiques. Le site de "La Caillouerie " pourrait aller dans le sens de cette dernière hypothèse, où le besoin consisterait au traitement 
de restes fauniques, voire même combiné à celui de la fabrication d'un certain nombre de supports lithiques voués à être transportés sur le site d'habitat.

Enfin, l'attention doit également se porter sur l'environnement très particulier du site, localisé sur un cordon littoral, à la jonction d'un marais palustre et de l'estran : les conditions peuvent difficilement être celles d'une zone d'habitat. D'ailleurs, l'absence de structures de type foyer, de bâtiments, le peu de mobilier céramique et la spécialisation de la production lithique sur ce site semblent indiquer cela (bien qu'il faille tenir compte de la faible ampleur de la fouille...). Par contre, la situation du gisement à l'embouchure du bassin de la Grise est favorable au développement d'une activité de pêche orientée vers la capture des espèces amphibiotiques (saumon, anguille). Les vestiges d'une pêcherie du $\mathrm{x}^{\mathrm{e}}$ siècle ont été reconnus à quelques centaines de mètres plus à l'est du site de " La Caillouerie » et les mentions de pêcheries médiévales sont présentes de manière ininterrompue jusqu'à la période moderne (Billard et al., 2006). Si le site n'a pas livré de restes de poissons, on peut tout de même s'interroger sur une relation possible entre une activité de pêche et l'assemblage lithique. Les pièces esquillées auraient pu servir au façonnage d'outils de pêche (en os?), tandis que les éclats auraient pu être impliqués dans la préparation des poissons (?). Le faible investissement technique pourrait dès lors être compensé par une bonne adaptation des produits lithiques à une activité spécifique.

\section{Perspectives}

Ce travail a permis de montrer l'intérêt de la tracéologie dans la détermination des pièces esquillées comme outils, ce que l'étude macroscopique ne permet pas complètement. En effet, si d'un point de vue typologique, une pièce esquillée peut à la fois désigner un outil et un nucléus, seul un croisement des données technologiques et tracéologiques, combiné à l'étude du mobilier associé et du contexte archéologique, permettent de trancher et d'émettre des hypothèses adaptées selon le site concerné (Lucas et Hays, 2004).

De plus, la découverte de « La Caillouerie » interroge sur la présence de site de l'âge du Bronze ancien en contexte littoral que la seule présence de lithique ne permet pas d'attribuer sans aucun doute à cette période chronologique. Ici, c'est bien la présence conjointe d'une céramique caractéristique combinée à des datations par le radiocarbone qui a permis de faire le lien.

Enfin, dans des conditions plus favorables à l'identification d'une activité spécialisée en relation avec les ressources littorales (conservation des restes de coquillages et de poissons), il sera certainement possible d'asseoir plus sûrement certaines hypothèses avancées.

\section{Bibliographie}

Bailly M., 2002 - «Du Néolithique à l'âge du Bronze ancien en Bassin Rhodanien. Une première approche du statut des productions lithiques ", in Mergorl M. (éd.), Les industries taillées holocènes $d u$ bassin rhodanien : problèmes et actualités, Actes de la table ronde de Lyon, 8-9 décembre 2000, collections Préhistoire, p. 205-223.

Bailly M., 2005 - "Production lithique domestique et débitage expédient dans le troisième millénaire de l'arc jurassien. Limites et contresens d'une qualification a priori ", in AsTruC L. (éd.), Au delà de la notion de technologie expédiente. Outillages lithiques au Néolithique, Actes de la table ronde du 15 mars 2004, Nanterre, Maison de l'Archéologie et de l'Ethnologie (Cahier des thèmes transversaux ArScAn, V), p. 201-209.

Bardon L., Bouyssonie A. et Bouyssonie J., I 906 - "Outils écaillées par percussion à la Coumba del Bouitou (Corrèze) ", Revue de l'école d'anthropologie, t. XVI, p. 170-175.

Besse M., 2003 - « Les céramiques communes des Campaniformes européens ", Gallia préhistoire, t. 45, p. 205-258.

Billard C., Bourhis J.-R., Desfossés Y., Evin J., Huault M.-F., Lefebvre D. et Paulet-Locard M.-A., I99I - «I. L'habitat des Florentins à Val-de-Reuil (Eure) », Gallia préhistoire, t. 33, p. 140-171.

Billard C., Aubry B., Blancquaert G., Bourhis J.-M., Habasque G., Marinval P., Pinzl C. et Ropars A., i994 « Poses-Le Vivier-Le Clos-Saint-Quentin (Eure) : l'occupation de la plaine inondable au Néolithique et au début de l'âge du Bronze ", Revue archéologique de l'Ouest, n 11, p. 53-113.

Billard C., Clet-Pellerin M. et Lautridiou J.-P., i 995 "Un site protohistorique littoral dans le havre de la Vanlée à Lingreville et Bricqueville-sur-Mer (Manche) ", Revue archéologique de l'Ouest, $\mathrm{n}^{\circ} 12$, p. 73-110.

Billard C., Barros J. et Laisné G., 2006 - « Portbail (Manche) : Nouvelles données sur les sites littoraux et l'évolution du havre de Portbail ", in L'exploitation des milieux littoraux en BasseNormandie, Programme Collectif de Recherche, Année 2006, SRA Basse-Normandie, Caen, p. 24-29.

Billard C., Bernard V., Bouffigny A., Clavel B., Couturier Y., Jaouen G., Le Digol Y., Quévillon S. et Ropars A., sous PRESSE - «Techniques et modes d'exploitation des pêcheries sur le littoral normand (France) : un essai de bilan de dix années de travaux archéologiques ", in Daire M.-Y., Dupont C., Baudry A., Billard C., Large J.-M., Lespez L., Normand E. et Scarre C. (dir.), Anciens peuplements littoraux et relations homme/milieu sur les côtes de l'Europe atlantique, Actes du colloque HOMER 2011, Vannes, 28 septembre - $1^{\text {er }}$ octobre 2011, Oxford : Archaeopress, British Archaeological Reports, International Series.

Boiral M. et Joussaume R., I 986 - " Sites campaniformes à La Tranche-sur-Mer (Vendée) ", in Joussaume R. (dir.), Cultures 
campaniformes dans le Centre-Ouest de la France, La Rochesur-Yon : groupe vendéen d'études préhistoriques, p. 67-76.

Bouchet J.-M., Burnez C., Roussot-Larroque J. et Villes A., I 990 - «Le Bronze ancien de la vallée de la Seugne : La Palut à Saint-Léger (Charente-Maritime) ", Gallia préhistoire, t. 32, p. 237-273.

Bougeant P., 2009 - "L'assemblage lithique recueilli sur le site campaniforme de l'Écuissière ", in LAPORTE L. (dir.), Des premiers paysans aux premiers métallurgistes sur la façade atlantique de la France (3500-2000 av. J.-C.), Chauvigny : association des publications chauvinoises, p. 411-412.

BRIARD J., I 970 - «Un tumulus du Bronze ancien : Kernonen en Plouvorn (Finistère) ", L'Anthropologie, t. 74, p. 5-56.

Briard J. et L'Helgouac'H J., I957 - Chalcolithique, Néolithique secondaire, survivances néolithiques à l'âge du Bronze ancien en Armorique, Travaux du laboratoire d'anthropologie et des musées préhistoriques de la faculté des sciences de Rennes, $72 \mathrm{p}$.

Chancerel A. et Masson B., I99 - « II. Le site du Clos-Bâtard à Tourville-la-Rivière (Seine-Maritime) ", Gallia préhistoire, t. 33, p. 172-184.

DAvidson D.-S., I 934 - "Australian spear-traits and their derivation ", Journal of the Polynesian Society, vol. 63, p. 41-72, 143-162.

Delahaye F., 2007 - Granville, cours Jonville, rapport de diagnostic archéologique, Inrap Grand-Ouest, SRA Basse-Normandie, Caen.

Dewez M., I985 - "Les pièces esquillées dans le Paléolithique supérieur de Belgique ", Bulletin de la société préhistorique française, t. 82, no 5, p. 131-133.

Donnart K, 2007 - Première approche diachronique du macrooutillage dans le Massif armoricain : du Néolithique moyen au début de l'âge du Bronze, Mémoire de Master 2, Université de Rennes 1, Rennes, 27 p.

Donnart K., 20 I 2 - «Une économie en grève : les matériaux du macro-outillage à la transition néolithique - âge du Bronze sur la côte ouest de la France ", in MéLin M. et Mougne C. (dir.), L'Homme, ses ressources et son environnement, dans le Nord-Ouest de la France à l'âge du Bronze : actualités de la recherche, actes du Séminaire archéologique de l'Ouest, 22 mars 2012, Rennes, Mémoires Géosciences Rennes, hors série nº 8, p. 71-89.

Donnart K., Naudinot N. et Le Clézio L., 2009 - « Approche expérimentale du débitage bipolaire sur enclume : caractérisation des produits et analyse des outils de production ", Bulletin de la société préhistorique française, t. 106, nº 3, p. 517-533.

Furestier R., 20II - "La percussion directe dure au Campaniforme : modalités d'application et difficultés d'interprétation ", in Mourre V. et JARry M. (dir.), "Entre le marteau et l'enclume... ", Actes de la table ronde de Toulouse, 15-17 mars 2004, PALEO, Numéro spécial 2009-2010, p. 189-200.
Gardez H., I935 - "Survivance de la pierre à l'âge du Bronze ", Bulletin de la société préhistorique française, t. 32, n 2, p. 147-148.

Ghesquière E., Marcigny C. et Rougier R., I 996 - «L'enceinte chalcolithique de "La plaine d'Ectot " à Ancrétiéville-SaintVictor (Seine-Maritime) ", Bulletin de la société préhistorique française, t. 93, $\mathrm{n}^{\circ} 1$, p. 97-102.

Gomez de Soto J., I 982 - "Céramique à pastillage, cordon en arceau, décor cordé vers une nouvelle approche du Bronze ancien dans le Centre-Ouest de la France ", Bulletin de la société préhistorique française, t. 79, p. 424-438.

Guyodo J.-N., 200 I - «Les assemblages lithiques des groupes néolithiques sur le Massif armoricain et ses marges ", Rennes : thèse de doctorat, université de Rennes I, 466 p.

Guyodo J.-N. et Marchand G., 2005 - « La percussion bipolaire sur enclume dans l'Ouest de la France de la fin du Paléolithique au Chalcolithique : une lecture économique et sociale ", Bulletin de la société préhistorique française, t. 102, no 3, p. 539-549.

Hayden B., I980 - "Confusion in the bipolar world: bashed pebbles and splintered pieces ", Lithic Technology, vol. 9, $\mathrm{n}^{\circ} 1$, p. $2-7$.

Hinguant S., Guyodo J.-N. et Hamon G., 2007 - «Une occupation littorale campaniforme à Saint-Malo (Ille-et-Vilaine) ", in Agogué O., Leroy D., Verjux C. (dir.), Camps, enceintes et structures d'habitat néolithiques en France septentrionale, Actes du $24^{\mathrm{e}}$ colloque interrégional sur le Néolithique, Orléans, 19-21 novembre 1999, 27e supplément à la Revue archéologique du Centre de la France, p. 309-326.

Joussaume R., I98 I - Le Néolithique de l'Aunis et du Poitou occidental dans son cadre atlantique, Rennes: Université Rennes I, travaux du laboratoire d'anthropologie, préhistoire, protohistoire et quaternaire armoricain, $625 \mathrm{p}$.

Laroche M. 201 2- Le gisement 18 Passe de l'Écuissière à Dolusd'Oléron (Charente-Maritime). Une occupation du Bronze ancien en milieu littoral, Rapport final d'opération, fouille préventive CCNBT, mars-avril 2011, 2 volumes, 224 p et 251 p.

Le Bihan J.-P., I 993 - Un habitat de transition Néolithique âge du Bronze, Quimper "Penancreac'h" (29.232.048.AH) Finistère, Document final de synthèse de sauvetage urgent, 15-02-1993 au 30-06-1993, Rennes, DRAC-SRA Bretagne, 62 p. + annexes.

Le Brun-Ricalens F., 2006 - "Les pièces esquillées : état des connaissances après un siècle de reconnaissance ", PALEO, t. 18, p. 95-114.

Le Roux C.-T., Lecerf Y. et Gautier M., i 983 - « Les alignements du moulin à Saint-Just avant, pendant et après l'érection des menhirs ", in Enclos funéraires et structures d'habitat en Europe du Nord-Ouest, table ronde du CNRS, Rennes 1981, travaux du laboratoire anthropologie, préhistoire, protohistoire, Quaternaire armoricains, Rennes, p. 121-134. 
LetTerlé F. et Verron G., I986 - «Un site d'habitat campaniforme à Digulleville (Manche), actes du $\mathrm{X}^{\mathrm{e}}$ colloque interrégional sur le Néolithique (Caen, 30 septembre - 2 octobre 1983) ", Revue archéologique de l'Ouest, suppl. 1, p. 237-252.

L'Homer A., I 995 - «Les vestiges de la pêcherie en bois de SaintJean-le-Thomas datant de l'âge du Bronze ", in Langouet L. et Morzadec-Kerfourn M.-T. (dir.), Baie du Mont-Saint-Michel et Marais de Dol, dossier du centre régional d'archéologie d'Alet, Saint-Malo, p. 119-124.

Longuet D., Péridy P. et Rouzeau N., I985 - « Le site campaniforme de la pointe de Grosse Terre, commune de Saint-Hilairede-Riez (Vendée) ", Études préhistoriques et protohistoriques, Pays de Loire, vol. 8, p. 31-42.

Lотн J., I 908 - "Remarques sur les pointes de flèche en silex à ailerons et pédoncule en Armorique ", Bulletin de la société polymathique du Morbihan, t. 50, p. 41-56.

LuCAs G. et Hays M., 2004 - « Les pièces esquillées du site paléolithique du Flageolet I (Dordogne) : outils ou nucléus? ", in Bodu P. et Constantin C. (éd.), Approches fonctionnelles en préhistoire, $\mathrm{XXV}^{\mathrm{e}}$ congrès préhistorique de France (2426 novembre 2000), Nanterre, p. 107-120.

Marcigny C. et Ghesquière E. (dir.), 2003 - L'île Tatihou (Manche) à l'âge du Bronze. Habitats et occupation du sol, DAF $\mathrm{n}^{\circ}$ 96, Paris, éd. de la Maison des Sciences de l'Homme, 192 p.

Marcigny C., Ghesquière E. et Gaumé É., 200 I - «Un habitat de l'âge du Bronze ancien à Guilberville "Le Domaigne " (Manche) ", Bulletin de la société préhistorique française, t. 98, $\mathrm{n}^{\circ} 3$, p. 529-541.

Marcigny C., Ghesquière E., Gaumé É., Giazzon D. et Gallouin E., 2003 - " Parcellaire et nécropoles de l'âge du Bronze ancien à Bernières-sur-Mer (Calvados) ", Bulletin de la société préhistorique française, t. 100, $\mathrm{n}^{\circ} 1$, p. 117-134.

Mazıère G., I984 - "La pièce esquillée, outil ou déchet?", Bulletin de la société préhistorique française, t. 81, nº 6, p. 182187.

Meurisse M., 2007 - «Enregistrement haute résolution des massifs dunaires Manche, mer du Nord et Atlantique : le rôle des tempêtes ", Lille : thèse de doctorat, Université Lille 1, 306 p.

Mortillet A. (de), i920 - "Les belles pointes de flèche en silex des sépultures morgiennes de Bretagne ", Bulletin de la société préhistorique française, t. XVII, p. 248-255.

Nicolas C., 2012 - "Des pierres précieuses? Les pointes de flèches du campaniforme et de l'âge du Bronze ancien en Bretagne (2500-1700 avant notre ère) ", in Marchand G. et
Querré G. (dir.), Roches et sociétés de la préhistoire entre massifs cristallins et bassins sédimentaires, Rennes, PUR, p. 205-219.

Pailler Y. et Dupont C. avec la collaboration de Sparfel Y. et Leroy A., 2007 - «Analyse fonctionnelle des galets biseautés du Mésolithique à la fin du Néolithique dans l'Ouest de la France, la Grande-Bretagne et l'Irlande ", Bulletin de la société préhistorique française, t. 104, $\mathrm{n}^{\circ} 1$, p. 31-54.

Pailler Y., Gandois H. et Tresset A. (dir.), 20 i I - Programme archéologique molénais, rapport no 17, Beg ar Loued: un habitat en pierres sèches campaniformelâge du Bronze ancien, fouille programmée du site de Beg ar Loued (île Molène; Finistère), opération no 2011-222, rapport de fouille, Rennes : SRA Bretagne, 2 volumes, 121 p. et 73 p.

Pautreau J.-P. et Robert P.-P., I980 - " Le gisement campaniforme des Deux Moulins au Bois-en-Ré (CharenteMaritime) ", Bulletin de la société préhistorique française, t. 77, $\mathrm{n}^{\circ}$ 9, p. 283-288.

Peña Alonso P. (de la), 20 i I - « Sobre la identificación macroscópica de las piezas astilladas: propuesta experimental ", Trabajos de Prehistoria, 68, n 1, 2011, p. 79-98.

Philippe J., I 927 - "Cinq années de fouilles au Fort-Harrouard 1921-1925 », Rouen, Lecerf, 176 p.

Poissonnier B., I 990 - "La métallurgie chalcolithique en Vendée ", in Vital C., Joussaume R. et Bernard E (éd.), 150 années de découvertes archéologiques en Vendée, la mort et le sacré, catalogue d'exposition, éd. L'Albaron, p. 78-80.

Querré G., 2009 - "Métallurgie et hautes températures Campaniforme et Artenac ", in LAPORTE L. (dir.), Des premiers paysans aux premiers métallurgistes sur la façade atlantique de la France (3500-2000 av. J.-C.), Chauvigny : association des publications chauvinoises, p. 540-541.

Rozor J.-G., I 978 - « Les derniers chasseurs. L'Épipaléolithique en France et en Belgique. Essai de synthèse ", Bulletin de la société archéologique champenoise, $\mathrm{n}^{\circ}$ spécial, 3 tomes, $1500 \mathrm{p}$.

Sorrel P., Debret M., Billeaud I., Jaccard S.-L., McManus J.-F. et Tessier B., 2012 - "Persistent non-solar forcing of Holocene storm dynamics in coastal sedimentary archives ", Nature geoscience, vol. 5, p. 892-896.

VIAu Y. (dir.), 2006 - Beauvoir-sur-Mer (Vendée), Le Pontreau 2. Fouille préventive $n^{\circ}$ 2004-205, Rapport final d'opération, Inrap Grand-Ouest, SRA Pays de la Loire, Nantes, 279 p.

VIAu Y. (dir.), 20 Io - Ancenis, Zac de la Savinière zone 5, un village ligérien du Bronze à la Tène, vol. 1, Rapport final d'opération, Inrap Grand-Ouest, SRA Pays de la Loire, Nantes, 358 p. 
Zusammenfassung: Der älterbronzezeitliche Fundplatz "La Caillouerie” von Saint-Lô d'Ourville (Manche), ein spezialisierter Fundplatz im Küstenbereich? - Die archäologische Fundstelle von "La Caillouerie" bei Saint-Lô-d'Ourville befindet sich im Hafen von Portbail. Sie wurde zufällig entdeckt, bedingt durch die fortschreitende Erosion des Strandbereichs in den Jahren 1990-2000. Die Fundstelle, die an den Anfang der frühen Bronzezeit datiert werden kann, hat eine bedeutende Menge an Flintartefakten geliefert, die von einer räumlich sehr begrenzten Fläche stammt. Die Analyse dieses umfangreichen Feuersteinmaterials hat es gestattet, einen Schlagplatz zu rekonstruieren, an dem kleine Flintknollen mit Hilfe bipolarer Abschlagstechnik bearbeitet wurden. Das Auftreten zahlreicher Flintartefakte stimmt aus typologischer Sicht gut mit den Flintabsplissen sowie mit dem Vorhandensein von intentionell erzeugten Knochensplittern tierischer Herkunft überein. Diese offensichtliche Übereinstimmung hat dazu geführt, eine Gebrauchsspurenanalyse an den Flintartefakten durchzuführen, um die Hypothese einer technologischen Verbindung zwischen diesen Fundobjekten zu bestätigen oder zu widerlegen. Die Untersuchung hat ergeben, dass nur ein bestimmter Teil dieser Flintartefakte tatsächlich benutzt wurde, um Langknochen aufzuspalten. Diese Fundstelle hat es gestattet, neue Ergebnisse zur Flintproduktion zu Beginn der Bronzezeit zu erzielen und ihre Bedeutung im Rahmen küstengebundener Rohstoffnutzung zu hinterfragen. Die neuen Ergebnisse lassen es ebenfalls zu, die Hypothese von spezialisierten Werkplätzen aufzustellen, die sich abseits von den üblichen Siedlungen etabliert haben.

Resumen: El sitio de la Edad de Bronce Antigua de "La Caillouerie" en Saint-Lô-d'Ourville (Manche): un sitio especializado en contexto costero? - El sitio de "La Caillouerie" en Saint-Lô-d'Ourville, situado en el puerto de Portbail, fue descubierto por casualidad después de una erosión importante de las playas del sector en los años 1990/2000. Fechado en el comienzo de la Edad del Bronce Antigua, entregó una gran cantidad de herramientas líticas en una superficie pequeña. El estudio de esta serie ha contribuido a destacar un taller de talla de pequeños guijarros costeros por medio de la técnica de la percusión sobre yunque. La presencia de muchos artefactos que corresponden tipológicamente a pedazos astillados asociados con elementos faunisticos intencionalmente fracturados, nos ha llevado a realizar un estudio traceológico para validar o invalidar la hipótesis de una relación entre estos diversos elementos. Este estudio mostró que sólo una parte de estas piezas habia sido utilizada en realidad como componentes intermedios para dividir los huesos largos. Este sitio permite traer nuevos conocimientos sobre las producciones líticas en los principios de la Edad del Bronce, y cuestionar su papel en la explotación del medio ambiente costero. También permite proponer la hipótesis de la presencia de talleres especializados probablemente fuera del ámbito doméstico.

Schlüsselwörter: Frühe Bronzezeit, Flintindustrie, Schlagplatz, Flint, Absplisse, bipolare Abschlagstechnik, küstengebundene Flintvorkommen, Gebrauchsspurenanalyse, Küste, Kanalküste, Knochensplitter, Keramik mit bogenförmigem Henkel

Palabras clave: Edad de Bronce antigua, industria litica, conjuntos de talla, silex, pedazos astillados, percusión sobre yunque, guijarros costeros, traceología, litoral, Manche, huesos divididos 